\title{
Prenatal and perinatal exposure of acrylamide disrupts the development of spinal cord in rats
}

\author{
Ahlam M. El-Bakry, M. Abdul-Hamid, A. Allam \\ Zoology Department, Faculty of Science, Beni-Suef University, Beni-Suef, Egypt \\ Email: amalbakry2@yahoo.com
}

Received 8 October 2012; revised 19 November 2012; accepted 15 December 2012

\begin{abstract}
Acrylamide is neurotoxic to the experimental animals and humans. Also, it has mutagenic and carcinogenic effects. Acrylamide was orally administered to nonanesthetized pregnant females by gastric intubation $(10 \mathrm{mg} / \mathrm{kg} / \mathrm{day})$. The animals were divided into three groups as follows: 1) Group A, newborn from control animals; 2) Group B, newborns from mothers treated with acrylamide from day 7 (D7) of gestation till birth (prenatal intoxicated group); 3) Group C, newborns from mothers treated with acrylamide from $D 7$ of gestation till D28 after birth (perinatally intoxicated group). In the present study acrylamide-induced histopathological and histochemical changes in brachial and lumber regions of spinal cord, including some pyknotic neurons and marked decrease of colour intensity of DNA contents as well as obvious retardation of sensorimotor reflexes of rat newborns. Thus acrylamide and its toxic metabolites resulted in teratogenicity in the rat newborns if their mother exposed to them chronically during gestation and lactation periods. As the spinal motor neurons are the final output neurons of motor systems, so a detailed developmental study is important for a greater understanding motor reflexes development. Moreover, the data on the acrylamide-induced effects on the embryonic and postnatal development is relatively rare. So, the present study was carried out to examine its effects on the development of spinal cord.
\end{abstract}

Keywords: Acrylamide; Spinal Cord; Anatomy; Histology; Development

\section{INTRODUCTION}

Acrylamide is a useful industrial agent used in the manufacture of polymers and synthetic organic chemicals. Polymeric acrylamide is used as filtration and flocculation aids in the water treatment and waste processing industries, mining and paper mills [1]. Acrylamide is metabolized to glycidamide by cytochrome P450 (CYP450)
[2-5]. Acrylamide interacts with glutathione-S-transferase (GST) $[2,6]$ to form $\mathrm{N}$-acetyl-S-cysteine which accounts approximately $70 \%$ of the urinary metabolites in the rat [7].

Acrylamide is a well-documented neurotoxicant that produces axonal pathology both in the central and peripheral nervous system [8-10]. Most of studies on acrylamide were done using adult rodents, and not much information is available on its effect on developing brain. During maturation of the brain, there is an extensive reorganization of neuronal structures, and exposure to neurotoxicant such as acrylamide would likely affect the expression of proteins, enzymes, lipids and nucleic acids.

The spinal motor neurons are the final output neurons of motor systems, so a detailed developmental study is important for a greater understanding motor reflexes development [11]. Neurophysiological research showed that the spinal cord has an intrinsic capacity for generating complex locomotor patterns in response to simple tonic inputs $[12,13]$. Motorneurons dysfunction due to spinal cord injury is a disastrous complication in humans [14]. Impaired reflex behaviors have been described in patients with spastic gait resulting from spinal cord injury [15]. Also, it is believed that the primary cause of the spinal muscular atrophy is motorneuron degeneration, while muscle weakness and atrophy occur secondarily $[16,17]$. Because the spinal motor neurons innervating limb muscles are the final output neurons of motor system, it is speculated that motor development in a rostrocaudal manner is closely related to the developmental changes of spinal motor neurons [18].

\section{Sensorimotor Reflexes}

The term reflex is a stereotyped and distinct neural response, which occurring largely at spinal level, evoked by a fixed and usually peripheral stimulus [19]. These peripheral sensory inputs modify the behavior of various animals during many different behaviors [20,21]. Impaired reflex input can contribute to severe dysfunction during human walking [19]. [22] recorded that the simplest 
motor actions are reflexes, which mediated by relatively straightforward circuitry in the spinal cord. The appearance and the maturation of a number of sensorimotor reflexes are components of the mature motor repertoire of the animal, and the expression of these can be correlated with the development and maturation of the nervous system [23]. [24] studied the effect of early nutritional deprivation on reflex ontogeny and recorded that the development of physical features and reflexes was significantly retarded in the malnourished rats.

Acrylamide affects on CNS and behavioral measures in rats and also produces detectable effects on rotarod performance prior to the occurrence of other observable effects $[10,21,25,26]$. The lack of acrylamide effects on forelimb hang reflex time or open field activity. The authors suggested that motor deficits may be just starting to appear in rats at the time they were tested in rotarod. The performance of multi-model negative geotaxis and rotarod tasks requires the involvement of numerous central and peripheral nervous system components. Thus, acrylamide effects on these two tasks may impact a variety of systems and/or regions, such as muscle strength, response to fatigue and cerebellar functions. However there is only limited literature correlating behavioral abnormalities with early pathological findings and their evolution $[21,25,27]$. High dose of acrylamide exposure has been shown to affect the dopaminergic system [28]. However appropriate functioning of the vestibular system is essential for negative geotaxis and rotarod performance [10, $29,30]$.

\section{MATERIALS AND METHODS}

\subsection{Chemicals}

Acrylamide (99\% pure) and other chemicals were purchased from Sigma chemical Company (St Louis, MO, USA). All other chemicals used were of analytical grade.

\subsection{Animals and Dosing Schedule}

The study has used a total of sixty albino rats (Rattus norvegicus). Forty five mature virgin females and 15 mature males weighing 140 - $150 \mathrm{~g}$ were purchased from the Organization for Vaccine and Biological Preparations, (Helwan laboratory farms, Egypt). Animals were marked, housed 4 per cage and fed standard rodent pellet diet manufactured by the Egyptian Company for Oil and Soap. Tap water was given ad libitum. Daily examination of vaginal smear of each virgin female was carried out to determine the estrous cycle. Estrous females exhibited the presence of cornified cells in vaginal smear. Mating was done by overnight housing of 2 pro-esterous females with one male in separate cages. The presence of sperm in vaginal smear determined the D0 of gestation. Acry- lamide was dissolved in distilled water and orally administered to non-anesthetized pregnant rats by gastric intubation at a dose of $10 \mathrm{mg} / \mathrm{kg} /$ day.

The newborn babies were labeled into 3 groups as follows:

Group A: pregnant rats were given saline (Normal group).

Group B: pregnant rats were administered acrylamide from D7 of gestation till birth (prenatal intoxicated group).

Group C: pregnant rats were administered acrylamide from D7 of gestation till D28 after birth (perinatal intoxicated group).

\subsubsection{Sensorimotor Reflex [31]}

At birth, each mother housed with its newborns in large cage kept in a ventilated room at constant temperature on a 12:12 h light/ dark cycle. Thirty newborns from D2 to D28 from each group were used for the present study. Each test was conducted every day 5 times for a given reflex from D2 until the reflex was expressed to its adult level for 3 consecutive days. Six newborns from each group were tested for each day of age. The positive responses were recorded (scores of $0 / 5$ to $5 / 5$ ). The average score was calculated, converted to percentage and a reflex was described as being present or absent. The first day a reflex was observed at least once in at least one animal was considered as the day of its appearance. A reflex was considered stable when it was expressed at the adult percentage for 3 consecutive days. The period between the appearance of a reflex and its stabilization was considered as the period of maturation. For testing, the animals were brought in a room $\left(25^{\circ} \mathrm{C}\right)$ reserved for that purpose also the reflex body righting in the air, the testing surface consisted of a slightly rough piece of Carton $(40 \mathrm{~cm} \times 60 \mathrm{~cm})$ of a light brown color. All tests were conducted by the same experimenter.

\subsubsection{Rooting}

The animal was put on the test surface and the experimenter formed a cone with the fingers around the snout of the animal. The reflex was considered present when the animal followed the movement of the experimenter slowly withdrawing his hand.

\subsubsection{Grasp}

The animal was held in air, the sole of the hand or the foot was gently touched with the tip of a fine brush. The left and right sides were tested equally. The reflex was considered present when the animal closed stimulated hand or foot around the brush.

\subsubsection{Body Righting on a Surface}

The animal was held in a supine position, the dorsum of the head and of the trunk in contact with the test surface. 
The reflex was considered present after the experimenter had released his hold, the animal turned on its ventrum or limbs within 15 seconds.

\subsubsection{Body Righting in the Air}

The animal was held supine in the air at a distance of 30 $\mathrm{cm}$ from soft surface. The reflex was considered present when release the animal turned in the air and landed on its ventrum or limbs.

\subsubsection{Hopping}

The animal was held so that only one hand or foot touched the test surface; it was moved forward as the tested limb dragged on the surface. The left and right sides were tested equally. The reflex was considered present when the animal lifted its limb and hopped in the direction of the movement.

\subsubsection{Chin Tactile Placing}

The animal was held by posterior half of the body and the skin of the chin was gently rubbed on the edge of the test surface. The reflex was considered present when the animal lifted one (or both) fore limb (FL) and placed it on the surface.

\subsubsection{Visual Placing}

The animal was held by tail at $10 \mathrm{~cm}$ from the test surface and was slowly brought closer to it. The reflex was considered present when the animal raised its head and extended its arm toward the surface before the arms came into with the surface.

\subsection{Histopathological and Histochemical Preparation of Spinal Cord Regions}

Segments of different spinal cord region were fixed in $10 \%$ buffered formalin $(\mathrm{pH} \mathrm{7.4)}$ for 48 hours. The tissue was dehydrated in $70 \%, 80 \%, 90 \%$ and $95 \%$ ethyl alcohol and 2 changes of absolute alcohol followed by two changes of xylene. The tissue was impregnated in paraffin wax and then embedded in paraffin wax. Sections (5 $\mu \mathrm{m})$ were cut, de-waxed, hydrated and stained in Mayer's haemalum solution for $3 \mathrm{~min}$. The sections were stained in Eosin for one min, washed in tap water and dehydrated. Haematoxylin and Eosin stained sections were prepared according to the method of [32], Feulgen method was used for staining DNA [33], and toludine blue stain [34].

\section{Statistical Analysis}

The Statistical Package for the Social Sciences (SPSS for windows version 11.0; SPSS Inc., Chicago) was used for the statistical analyses. Comparative analyses were conducted by using the general linear models procedure
(SPSS, Inc.). The data were analyzed using one-way and two-way analysis of variance (ANOVA) followed by LSD computations to compare various groups with each other. F-values express the general effect between groups, and the effect of time, treatment and their interaction. Results were expressed as mean \pm S.D. The level of significance was expressed as $P>0.05$ for insignificantly different, while $P<0.05$ was significantly different, whereas $P<0.01$ and $\mathrm{P}<0.001$ were highly and very highly significantly different, respectively [35].

\section{RESULTS}

\subsection{Sensorimotor Reflexes}

The following reflexes; rooting, FL grasp, HL grasp, surface body righting, air body righting, FL hopping, HL hopping, chin tactile placing and visual placing were examined in the newborns of all groups from D2 until reflex maturation.

\subsubsection{Rooting}

Rooting was absent in $100 \%$ of all postnatal stages in all groups between D2 and D28.

\subsubsection{Grasp}

\subsubsection{Fore-Limb (FL) Grasp}

This reflex was absent in all groups at D2. It appeared at D4 by $31.67 \%$ in normal newborns and increased rapidly to reach $100 \%$ at D10. The pattern of the reflex curve indicated the high activation in these newborns. In Group B, it appeared at D4 by $8.33 \%$ and increased to reach $100 \%$ at D15. In Group C, it appeared at D5 by $15 \%$ and increased to attain $100 \%$ at D17. There were fluctuations in the activation of the newborns as shown by the reflex curve in Group B. Also, in Group C, the reflex curve showed its development by a low rate (Figure 1). The present analysis showed significant difference between normal and treated groups as shown in Table $\mathbf{1 .}$

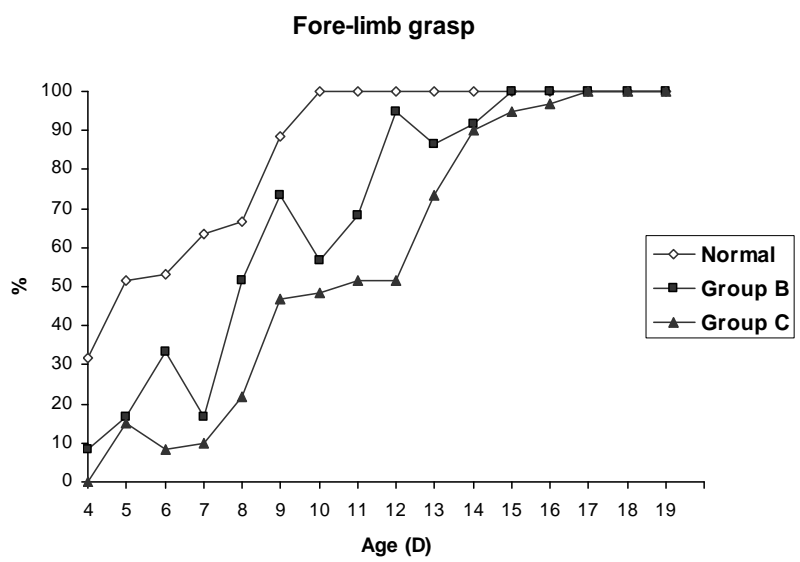

Figure 1. Fore-limb grasps percentage of rat newborns. 
Table 1. Ontogenesis of FL grasp reflex in rat newborns.

\begin{tabular}{cccc}
\hline D/Groups & A & B & C \\
\hline $\mathbf{4}$ & $31.67 \pm 3.06$ & $8.33 \pm 3.07^{* * *}$ & $0.0 \pm 0.0^{* * *}$ \\
$\mathbf{5}$ & $51.67 \pm 3.07$ & $16.67 \pm 5.58^{* *}$ & $15.00 \pm 4.28^{* * *}$ \\
$\mathbf{6}$ & $53.33 \pm 3.33$ & $33.33 \pm 5.58^{*}$ & $8.33 \pm 3.07^{* * *}$ \\
$\mathbf{7}$ & $63.33 \pm 3.33$ & $16.67 \pm 5.58^{* *}$ & $10.00 \pm 3.65^{* * *}$ \\
$\mathbf{8}$ & $66.67 \pm 3.33$ & $51.67 \pm 3.07^{*}$ & $21.67 \pm 5.43^{* * *}$ \\
$\mathbf{9}$ & $88.33 \pm 3.07$ & $73.33 \pm 4.94$ & $46.67 \pm 4.22^{* * *}$ \\
$\mathbf{1 0}$ & $100.0 \pm 0.00$ & $56.67 \pm 6.14^{* * *}$ & $48.33 \pm 5.43^{* * *}$ \\
$\mathbf{1 1}$ & $100.0 \pm 0.00$ & $68.33 \pm 3.07^{* * *}$ & $51.67 \pm 3.07^{* * *}$ \\
$\mathbf{1 2}$ & $100.0 \pm 0.00$ & $95.00 \pm 2.23$ & $51.67 \pm 3.07^{* * *}$ \\
$\mathbf{1 3}$ & $100.0 \pm 0.00$ & $86.67 \pm 4.21^{*}$ & $73.33 \pm 3.33^{* * *}$ \\
$\mathbf{1 4}$ & $100.0 \pm 0.00$ & $91.67 \pm 3.07^{*}$ & $90.00 \pm 2.58^{*}$ \\
$\mathbf{1 5}$ & $100.0 \pm 0.00$ & $100.0 \pm 0.00$ & $95.00 \pm 2.23$ \\
$\mathbf{1 6}$ & $100.0 \pm 0.00$ & $100.0 \pm 0.00$ & $96.67 \pm 3.33$ \\
$\mathbf{1 7}$ & $100.0 \pm 0.00$ & $100.0 \pm 0.00$ & $100.0 \pm 0.00$ \\
\hline
\end{tabular}

Data are expressed as a mean \pm S.E. $(N=6)$; Values significantly compared to control newborns; ${ }^{*} p \leq 0.05,{ }^{* *} p \leq 0.01$ and ${ }^{* * *} p \leq 0.001$.

\subsubsection{Hind-Limb (HL) Grasp}

In normal newborns, HL grasp appeared later than FL grasp and it was detected by $18.33 \%$ at D10 and increased to reach $100 \%$ at D16. The linear shape of the reflex curve of normal newborns showed the regularity in the development of this reflex. In Groups B and C, it appeared at D13 by $11.67 \%$ and $6.67 \%$, respectively and reached $100 \%$ at D19 and D21 in Groups B and C, respectively. In the latter group, the reflex development at the first two days of its expression was very slowly (Figure 2). The level of significance between normal and treated newborns showed some variability (Table 2).

\subsubsection{Surface Body Righting}

This precocious reflex was already expressed by $50 \%$ of the trials in Group A at D2 and increased quickly to reach $100 \%$ at D5. In Groups B and C this reflex appeared by $10 \%$ at $\mathrm{D} 2$ and its expression increased in Group B faster than in Group C to reach $100 \%$ at D12 and D13, respectively. The irregularities in the reflex curve indicate the weakness of the newborns of these groups (Figure 3). The difference between normal and treated groups was significant (Table 3).

\subsubsection{Air Body Righting}

In normal newborns, the reflex appeared by $30 \%$ at D11, then its expression increased regularly to attain $100 \%$ at D17. The development of air body righting reflex in treated groups reflected the retardation in reflex develop-

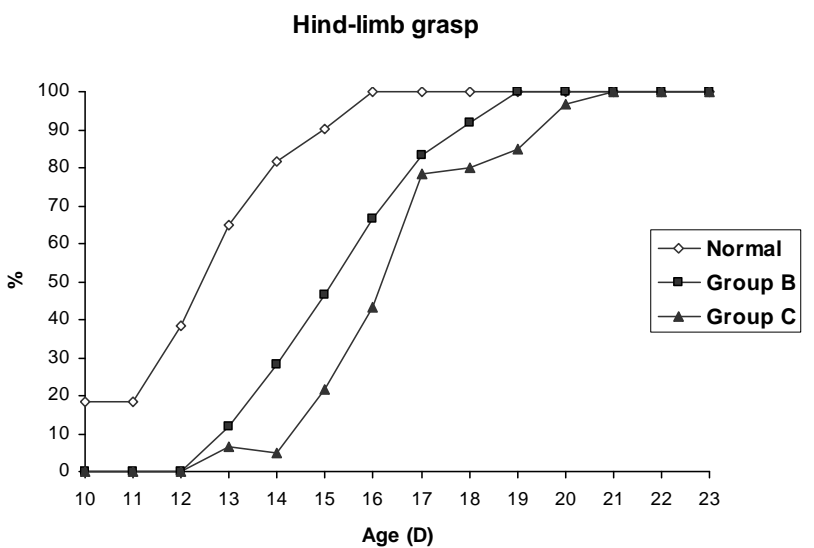

Figure 2. Hind-limb grasps percentage of different groups.

Table 2. Ontogenesis of HL grasp reflex in rat newborns.

\begin{tabular}{cccc}
\hline D/Groups & A & B & C \\
\hline $\mathbf{1 0}$ & $18.33 \pm 3.07$ & $0.0 \pm 0.0^{* *}$ & $0.0 \pm 0.0^{* *}$ \\
$\mathbf{1 1}$ & $18.33 \pm 3.07$ & $0.0 \pm 0.0^{* *}$ & $0.0 \pm 0.0^{* *}$ \\
$\mathbf{1 2}$ & $38.33 \pm 5.42$ & $0.0 \pm 0.0^{* * *}$ & $0.0 \pm 0.0^{* * *}$ \\
$\mathbf{1 3}$ & $65.00 \pm 2.24$ & $11.67 \pm 4.77^{* * *}$ & $6.67 \pm 3.33^{* * *}$ \\
$\mathbf{1 4}$ & $81.67 \pm 3.07$ & $28.33 \pm 6.00^{* * *}$ & $5.00 \pm 2.23^{* * *}$ \\
$\mathbf{1 5}$ & $90.0 \pm 3.65$ & $46.67 \pm 7.60^{* *}$ & $21.67 \pm 5.42^{* * *}$ \\
$\mathbf{1 6}$ & $100.0 \pm 0.00$ & $66.67 \pm 4.21^{* * *}$ & $43.33 \pm 6.15^{* * *}$ \\
$\mathbf{1 7}$ & $100.0 \pm 0.00$ & $83.33 \pm 4.21^{*}$ & $78.33 \pm 4.01^{* *}$ \\
$\mathbf{1 8}$ & $100.0 \pm 0.00$ & $91.67 \pm 3.07^{*}$ & $80.00 \pm 3.65^{* *}$ \\
$\mathbf{1 9}$ & $100.0 \pm 0.00$ & $100.0 \pm 0.00$ & $85.00 \pm 4.28^{*}$ \\
$\mathbf{2 0}$ & $100.0 \pm 0.00$ & $100.0 \pm 0.00$ & $96.67 \pm 2.11$ \\
$\mathbf{2 1}$ & $100.0 \pm 0.00$ & $100.0 \pm 0.00$ & $100 \pm 0.0$ \\
\hline
\end{tabular}

Data are expressed as a mean \pm S.E. $(N=6)$; Values significantly compared to control newborns; ${ }^{*} p \leq 0.05,{ }^{* *} p \leq 0.01$ and ${ }^{* * *} p \leq 0.001$.

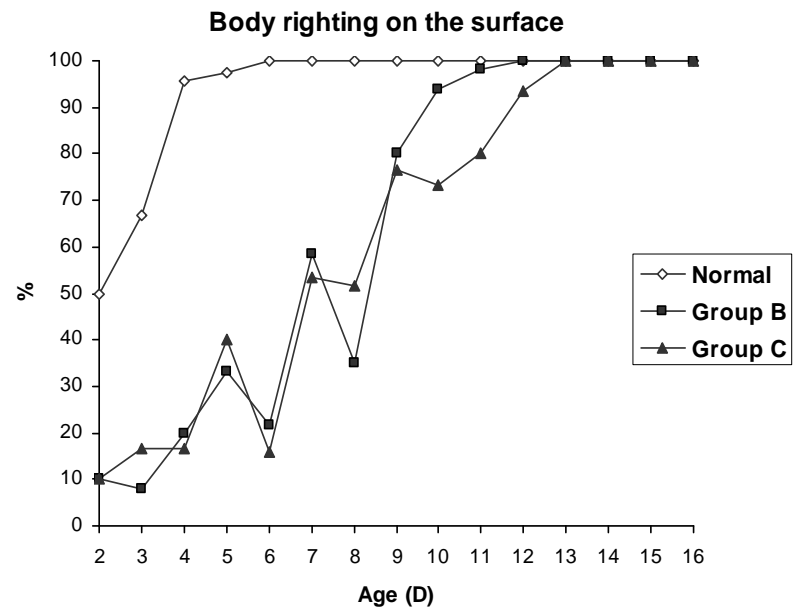

Figure 3. Body righting on the surface percentage of rat newborns. 
Table 3. Ontogenesis of surface body righting reflex in rat newborns.

\begin{tabular}{cccc}
\hline D\Groups & A & B & C \\
\hline $\mathbf{2}$ & $50.00 \pm 2.89$ & $10.00 \pm 2.58^{* * *}$ & $10.00 \pm 2.58^{* * *}$ \\
$\mathbf{3}$ & $66.67 \pm 3.78$ & $8.00 \pm 3.06^{* * *}$ & $16.67 \pm 6.67^{* * *}$ \\
$\mathbf{4}$ & $95.83 \pm 1.56$ & $20.00 \pm 5.77^{* * *}$ & $16.67 \pm 4.21^{* * *}$ \\
$\mathbf{5}$ & $97.50 \pm 1.23$ & $33.33 \pm 4.94^{* * *}$ & $40.00 \pm 6.83^{* * *}$ \\
$\mathbf{6}$ & $100.0 \pm 0.00$ & $21.67 \pm 7.92^{* * *}$ & $16.00 \pm 4.16^{* * *}$ \\
$\mathbf{7}$ & $100.0 \pm 0.00$ & $58.33 \pm 9.46^{* *}$ & $53.33 \pm 6.15^{* * *}$ \\
$\mathbf{8}$ & $100.0 \pm 0.00$ & $35.00 \pm 12.58^{* * *}$ & $51.67 \pm 4.77^{* * *}$ \\
$\mathbf{9}$ & $100.0 \pm 0.00$ & $80.00 \pm 5.77^{*}$ & $76.67 \pm 4.22^{* *}$ \\
$\mathbf{1 0}$ & $100.0 \pm 0.00$ & $94.00 \pm 3.27$ & $73.33 \pm 10.22^{*}$ \\
$\mathbf{1 1}$ & $100.0 \pm 0.00$ & $98.33 \pm 1.67$ & $80.00 \pm 3.65^{* *}$ \\
$\mathbf{1 2}$ & $100.0 \pm 0.00$ & $100.0 \pm 0.00$ & $93.33 \pm 4.22$ \\
$\mathbf{1 3}$ & $100.0 \pm 0.00$ & $100.0 \pm 0.00$ & $100.0 \pm 0.00$ \\
\hline
\end{tabular}

Data are expressed as a mean \pm S.E. $(N=6)$; Values significantly compared to control newborns; ${ }^{*} p \leq 0.05,{ }^{* *} p \leq 0.01$ and ${ }^{* * *} p \leq 0.001$.

ment where, it expressed at D15 by $16.67 \%$ in Group B and at D17 by $18.33 \%$ in Group C. The activation increased slowly to reach $100 \%$ at D20 in Group B and at D22 in Group C (Table 4 and Figure 4).

\subsubsection{Hopping}

\subsubsection{FL Hopping}

FL hopping appeared in normal newborns by $48.33 \%$ at D3 and its expression was regular and increased rapidly to attain $100 \%$ at D8. It was observed by $13.33 \%$ and $43.33 \%$ at D4 and D5 in Groups B and C, respectively. The reflex expression increased slowly with irregular rate to reach $100 \%$ at D14 in Group B and at D15 in Group C (Figure 5). Table 5 elucidates the level of significance between normal and treated groups.

\subsubsection{HL Hopping}

This reflex appeared in normal group by $38.33 \%$ at D3 and its expression increased quickly with regular pattern to reach $100 \%$ at D10. In Groups B and C, it was detected by $21.67 \%$ and $11.67 \%$, respectively at D5 and increased slowly to reach $100 \%$ at D16 in Group B and at D18 in Group C. The pattern of reflex curves of treated groups showed the abnormalities in Groups B and C compared with normal group (Figure 6). The level of significance between normal and treated groups was varied (Table 6).

\subsubsection{Chin Tactile Placing}

The expression of this reflex was detected in normal newborns by $8.33 \%$ at $\mathrm{D} 4$ then it was regular until it showed $100 \%$ at D12. Detection of this reflex in Groups
Table 4. Ontogenesis of air body righting reflex in rat newborns.

\begin{tabular}{cccc}
\hline D $\backslash$ Groups & A & B & C \\
\hline $\mathbf{1 1}$ & $30.00 \pm 3.65$ & $00.0 \pm 0.0^{* * *}$ & $00.0 \pm 0.0^{* * *}$ \\
$\mathbf{1 2}$ & $28.33 \pm 3.07$ & $00.0 \pm 0.0^{* * *}$ & $00.0 \pm 0.0^{* * *}$ \\
$\mathbf{1 3}$ & $31.67 \pm 3.07$ & $00.0 \pm 0.0^{* * *}$ & $00.0 \pm 0.0^{* * *}$ \\
$\mathbf{1 4}$ & $53.33 \pm 3.33$ & $00.0 \pm 0.0^{* * *}$ & $00.0 \pm 0.0^{* * *}$ \\
$\mathbf{1 5}$ & $80.00 \pm 3.65$ & $16.67 \pm 5.58^{* * *}$ & $00.0 \pm 0.0^{* * *}$ \\
$\mathbf{1 6}$ & $95.00 \pm 2.24$ & $16.67 \pm 8.43^{* * *}$ & $00.0 \pm 0.0^{* * *}$ \\
$\mathbf{1 7}$ & $100.0 \pm 0.00$ & $28.33 \pm 8.33^{* * *}$ & $18.33 \pm 3.07^{* * *}$ \\
$\mathbf{1 8}$ & $100.0 \pm 0.00$ & $68.33 \pm 3.07^{* * *}$ & $30.00 \pm 5.77^{* * *}$ \\
$\mathbf{1 9}$ & $100.0 \pm 0.00$ & $85.00 \pm 3.42^{* *}$ & $46.67 \pm 8.82^{* *}$ \\
$\mathbf{2 0}$ & $100.0 \pm 0.00$ & $100.0 \pm 0.00$ & $63.33 \pm 10.54^{*}$ \\
$\mathbf{2 1}$ & $100.0 \pm 0.00$ & $100.0 \pm 0.00$ & $95.00 \pm 3.42$ \\
$\mathbf{2 2}$ & $100.0 \pm 0.00$ & $100.0 \pm 0.00$ & $100.0 \pm 0.00$ \\
\hline
\end{tabular}

Data are expressed as a mean \pm S.E. $(N=6)$; Values significantly compared to control newborns; ${ }^{*} p \leq 0.05,{ }^{* *} p \leq 0.01$ and ${ }^{* * *} p \leq 0.001$.

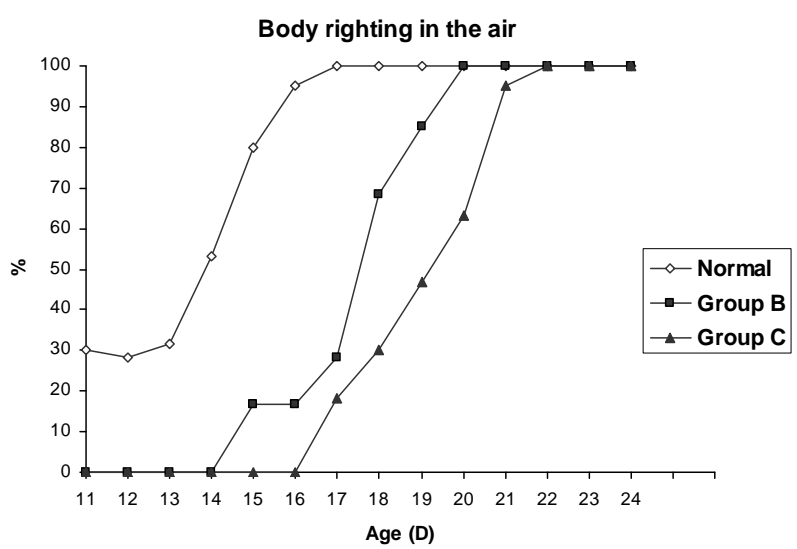

Figure 4. Body righting in the air percentage of rat newborns.

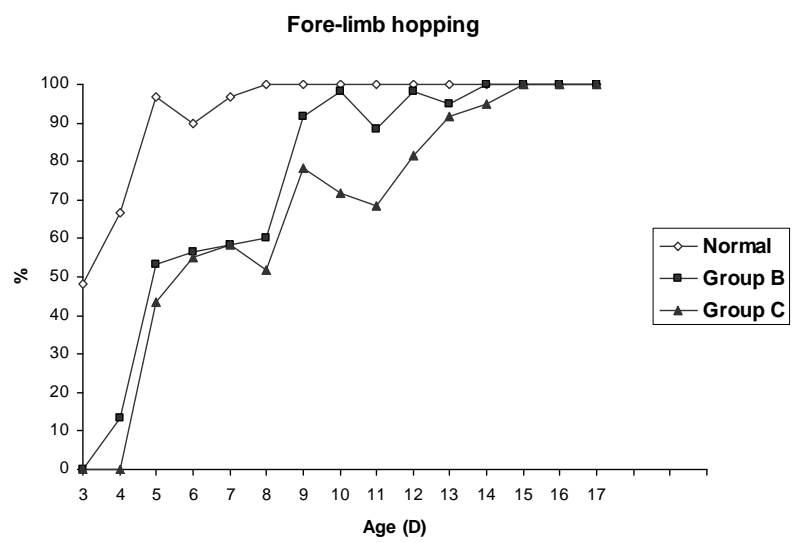

Figure 5. Fore-limb hopping percentage of rat newborns.

B and C noticed at D9 by $21.67 \%$ and $8.33 \%$, respectively then it increased rapidly to reach $100 \%$ in Group B at D17 and in Group C at D18 (Table 7 and Figure 7). 
Table 5. Ontogenesis of FL hopping reflex in rat newborns.

\begin{tabular}{cccc}
\hline D/Groups & A & B & C \\
\hline $\mathbf{3}$ & $48.33 \pm 3.07$ & $0.0 \pm 0.0^{* * *}$ & $0.0 \pm 0.0^{* * *}$ \\
$\mathbf{4}$ & $66.67 \pm 3.33$ & $13.33 \pm 4.22^{* * *}$ & $0.0 \pm 0.0^{* * *}$ \\
$\mathbf{5}$ & $96.67 \pm 2.11$ & $53.33 \pm 6.15^{* * *}$ & $43.33 \pm 4.22^{* * *}$ \\
$\mathbf{6}$ & $90.00 \pm 2.58$ & $56.67 \pm 7.60^{*}$ & $55.00 \pm 7.64^{*}$ \\
$\mathbf{7}$ & $96.67 \pm 5.16$ & $58.33 \pm 5.43^{* * *}$ & $58.33 \pm 6.01^{* *}$ \\
$\mathbf{8}$ & $100.0 \pm 0.00$ & $60.00 \pm 6.83^{* *}$ & $51.67 \pm 5.43^{* * *}$ \\
$\mathbf{9}$ & $100.0 \pm 0.00$ & $91.67 \pm 1.67^{* *}$ & $78.33 \pm 3.07^{* * *}$ \\
$\mathbf{1 0}$ & $100.0 \pm 0.00$ & $98.33 \pm 1.67$ & $71.67 \pm 4.77^{* *}$ \\
$\mathbf{1 1}$ & $100.0 \pm 0.00$ & $88.33 \pm 4.77$ & $68.33 \pm 4.77^{* *}$ \\
$\mathbf{1 2}$ & $100.0 \pm 0.00$ & $98.33 \pm 1.67$ & $81.67 \pm 3.07^{* *}$ \\
$\mathbf{1 3}$ & $100.0 \pm 0.00$ & $95.00 \pm 3.42$ & $91.67 \pm 3.07^{*}$ \\
$\mathbf{1 4}$ & $100.0 \pm 0.00$ & $100.0 \pm 0.00$ & $95.00 \pm 3.42$ \\
$\mathbf{1 5}$ & $100.0 \pm 0.00$ & $100.0 \pm 0.00$ & $100.0 \pm 0.00$ \\
\hline
\end{tabular}

Data are expressed as a mean \pm S.E. $(N=6)$; Values significantly compared to control newborns; ${ }^{*} p \leq 0.05,{ }^{* *} p \leq 0.01$ and ${ }^{* * *} p \leq 0.001$.

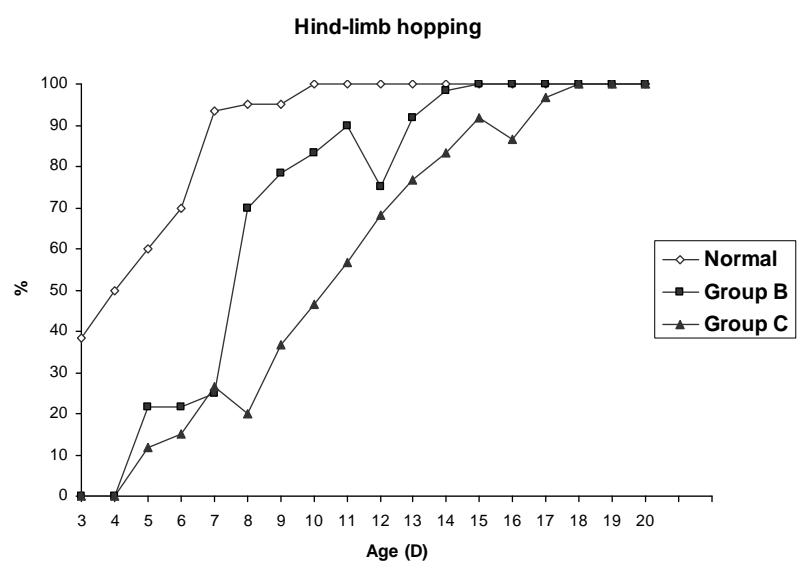

Figure 6. Hind-limb hopping percentage of rat newborns.

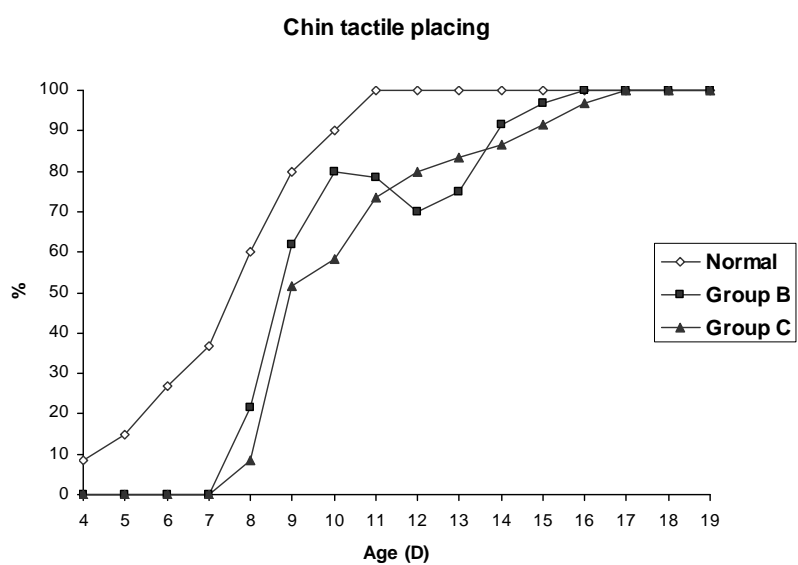

Figure 7. Chin tactile placing percentage of rat newborns.
Table 6. Ontogenesis of HL hopping reflex in rat newborns.

\begin{tabular}{|c|c|c|c|}
\hline $\mathrm{D} \backslash$ Groups & A & B & $\mathrm{C}$ \\
\hline 3 & $38.33 \pm 3.07$ & $0.0 \pm 0.0^{* *}$ & $0.0 \pm 0.0^{* * *}$ \\
\hline 4 & $50.00 \pm 3.65$ & $0.0 \pm 0.0^{* *}$ & $0.0 \pm 0.0^{* * *}$ \\
\hline 5 & $60.00 \pm 3.65$ & $21.67 \pm 3.07^{* *}$ & $11.67 \pm 3.07^{* * *}$ \\
\hline 6 & $70.00 \pm 3.65$ & $21.67 \pm 3.07^{* *}$ & $15.00 \pm 2.24^{* * *}$ \\
\hline 7 & $93.33 \pm 3.33$ & $25.00 \pm 4.28^{* * *}$ & $26.67 \pm 4.22^{* * *}$ \\
\hline 8 & $95.00 \pm 2.24$ & $70.00 \pm 8.56^{* *}$ & $20.00 \pm 5.77^{* * *}$ \\
\hline 9 & $95.00 \pm 3.42$ & $78.33 \pm 6.54^{* * *}$ & $36.67 \pm 7.60^{* *}$ \\
\hline 10 & $100.0 \pm 0.00$ & $83.33 \pm 4.22^{* * *}$ & $46.67 \pm 3.33^{* * *}$ \\
\hline 11 & $100.0 \pm 0.00$ & $90.00 \pm 2.58^{* * *}$ & $56.67 \pm 4.22^{* * *}$ \\
\hline 12 & $100.0 \pm 0.00$ & $75.00 \pm 4.28^{* * *}$ & $68.33 \pm 6.01^{* *}$ \\
\hline 13 & $100.0 \pm 0.00$ & $91.67 \pm 3.07^{* * *}$ & $76.67 \pm 2.11^{* * *}$ \\
\hline 14 & $100.0 \pm 0.00$ & $98.33 \pm 1.67$ & $83.33 \pm 4.22^{*}$ \\
\hline 15 & $100.0 \pm 0.00$ & $100.0 \pm 0.00$ & $91.67 \pm 1.67^{* *}$ \\
\hline 16 & $100.0 \pm 0.00$ & $100.0 \pm 0.00$ & $86.67 \pm 4.94^{*}$ \\
\hline 17 & $100.0 \pm 0.00$ & $100.0 \pm 0.00$ & $96.67 \pm 2.11$ \\
\hline 18 & $100.0 \pm 0.00$ & $100.0 \pm 0.00$ & $100.0 \pm 0.00$ \\
\hline
\end{tabular}

Data are expressed as a mean \pm S.E. $(N=6)$; Values significantly compared to control newborns; ${ }^{*} p \leq 0.05,{ }^{* * *} p \leq 0.01$ and ${ }^{* * *} p \leq 0.001$.

Table 7. Ontogenesis of chin tactile placing reflex in rat newborns.

\begin{tabular}{cccc}
\hline D/Groups & A & B & C \\
\hline $\mathbf{4}$ & $8.33 \pm 4.77$ & $0.0 \pm 0.0$ & $0.0 \pm 0.0$ \\
$\mathbf{5}$ & $15.00 \pm 5.63$ & $0.0 \pm 0.0^{*}$ & $0.0 \pm 0.0^{*}$ \\
$\mathbf{6}$ & $27.00 \pm 5.86$ & $0.0 \pm 0.0^{* *}$ & $0.0 \pm 0.0^{* *}$ \\
$\mathbf{7}$ & $36.67 \pm 3.33$ & $0.0 \pm 0.0^{* * *}$ & $0.0 \pm 0.0^{* * *}$ \\
$\mathbf{8}$ & $60.00 \pm 2.58$ & $21.67 \pm 4.77^{* * *}$ & $8.33 \pm 4.01^{* * *}$ \\
$\mathbf{9}$ & $80.00 \pm 2.58$ & $61.67 \pm 4.77^{*}$ & $51.67 \pm 3.07^{* * *}$ \\
$\mathbf{1 0}$ & $90.00 \pm 3.65$ & $80.00 \pm 5.77$ & $58.33 \pm 4.77^{* *}$ \\
$\mathbf{1 1}$ & $100.0 \pm 0.00$ & $78.33 \pm 4.77^{* *}$ & $73.33 \pm 4.22^{* * *}$ \\
$\mathbf{1 2}$ & $100.0 \pm 0.00$ & $70.00 \pm 3.65^{* * *}$ & $80.00 \pm 4.47^{* *}$ \\
$\mathbf{1 3}$ & $100.0 \pm 0.00$ & $75.00 \pm 4.28^{* *}$ & $83.33 \pm 6.67$ \\
$\mathbf{1 4}$ & $100.0 \pm 0.00$ & $91.67 \pm 3.07^{*}$ & $86.67 \pm 4.22^{*}$ \\
$\mathbf{1 5}$ & $100.0 \pm 0.00$ & $96.67 \pm 2.11$ & $91.67 \pm 3.07^{*}$ \\
$\mathbf{1 6}$ & $100.0 \pm 0.00$ & $100.0 \pm 0.00$ & $96.67 \pm 2.11$ \\
$\mathbf{1 7}$ & $100.0 \pm 0.00$ & $100.0 \pm 0.00$ & $100.0 \pm 0.00$ \\
\hline
\end{tabular}

Data are expressed as a mean \pm S.E. $(N=6)$; Values significantly compared to control newborns; ${ }^{*} p \leq 0.05,{ }^{* *} p \leq 0.01$ and ${ }^{* * *} p \leq 0.001$. 


\subsubsection{Visual Placing}

This late reflex appeared at D15 in normal group by $46 \%$ and matured rapidly to reach $100 \%$ at D17. It was detected in Groups B and C at D16 and D17 by $28.3 \%$ and $30 \%$, respectively then its expression increased to reach $100 \%$ at D19 in Group B and at D21 in Group C (Figure 8). This reflected the developmental retardation in eye opening besides to the inability of the newborns to express the reflex physically in the treated groups. Table 8 revealed the level of significance between normal and treated groups.

\subsection{The Histopathology and Histochemical Results of Brachial Region of Spinal Cord}

\subsubsection{Haematoxylin-Eosin Stain}

In the present rat newborns, the spinal cord was surrounded by meninges. The inner most layer of meninges is the pia matter which adheres closely to the superficial membranes of the cord. The second membrane is the arachnoid. Externally, there is a thick fibrous dura matter separated from the arachnoid by subdural space. The brachial spinal cord is consists of centrally located gray matter and a peripheral white matter. In the gray matter, the

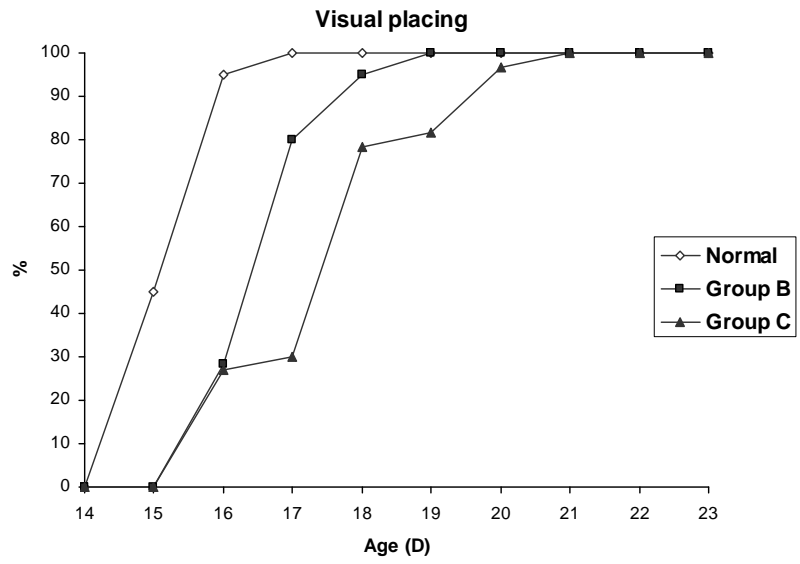

Figure 8. Visual placing percentage in rat newborns.

Table 8. Ontogenesis of visual placing reflex in rat newborns.

\begin{tabular}{cccc}
\hline D\Groups & A & B & C \\
\hline $\mathbf{1 5}$ & $45.00 \pm 5.63$ & $0.0 \pm 0.0^{* * *}$ & $0.0 \pm 0.0^{* * *}$ \\
$\mathbf{1 6}$ & $95.00 \pm 2.24$ & $28.33 \pm 10.14^{* * *}$ & $27.0 \pm 8.6^{* * *}$ \\
$\mathbf{1 7}$ & $100.0 \pm 0.00$ & $80.00 \pm 5.77^{*}$ & $30.00 \pm 10.33^{* * *}$ \\
$\mathbf{1 8}$ & $100.0 \pm 0.00$ & $95.00 \pm 2.24$ & $78.33 \pm 3.07^{* * *}$ \\
$\mathbf{1 9}$ & $100.0 \pm 0.00$ & $100.0 \pm 0.00$ & $81.67 \pm 3.07^{* *}$ \\
$\mathbf{2 0}$ & $100.0 \pm 0.00$ & $100.0 \pm 0.00$ & $96.67 \pm 2.11$ \\
$\mathbf{2 1}$ & $100.0 \pm 0.00$ & $100.0 \pm 0.00$ & $100.0 \pm 0.00$ \\
\hline
\end{tabular}

Data are expressed as a mean \pm S.E. $(N=6)$; Values significantly compared to control newborns; ${ }^{*} p \leq 0.05,{ }^{* *} p \leq 0.01$ and ${ }^{* * *} p \leq 0.001$. neurons and glial cells were distributed. The white matter has the axons of neurons and some glial (neuroglia) cells (Figure 9).

At D7, the normal motorneurons were large in size, had many processes and had oval nuclei (Figure 9(a)). In Group B, most motorneurons appeared small in size. Also, some pyknotic cells were noticed (Figure 9(b)). In Group C, motorneurons chromatolysis was recorded (Figure 9(c)). Between D14 and D28, the normal motorneurons increased in size with a more clearly differentiated chromatin and a distinct thick coat of cytoplasm (Fig-

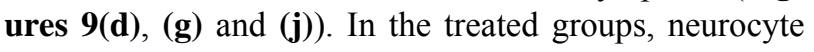

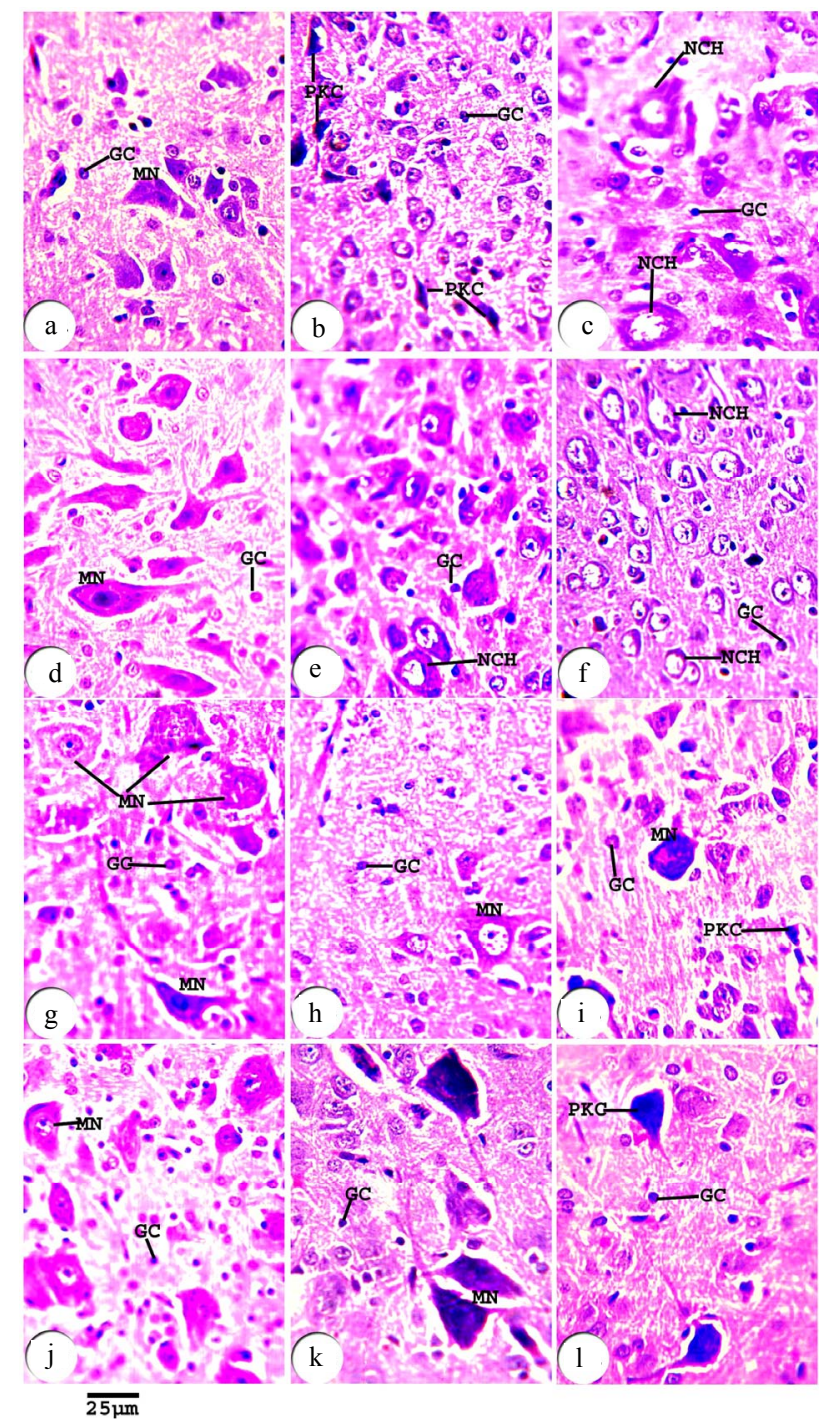

Figure 9. Transverse sections in the brachial spinal region showing the glial cells (GC), motorneurons (MN), neurocyte chromatolysis $(\mathrm{NCH})$ and pyknosis (PKC). (a) Normal group at D7; (b) Group B at D7; (c) Group C at D7; (d) Normal group at D14; (e) Group B at D14; (f) Group C at D14; (g) Normal group at D21; (h) Group B at D21; (i) Group C at D21; (j) Normal group at D28; (k) Group B at D28 and (1) Group C at D28 (H. \& E.). 
chromatolysis and pyknotic neurons were present (Figures 9(e), (f), (i) and (l)). Group B showed some improvement at D21 and D28 (Figures 9(h) and (k)).

\subsubsection{Toludine-Blue Stain}

During the growth, Nissl granules were found in the perikaryon and in the proximal parts of the dendrites of the neurons. At D7, the normal motorneurons stained well so, the intensity of Nissl granules was high (Figure 10(a)). In Groups B and C motorneurons were paled stained (Figures 10(b) and (c)).

Between D14 and D28, the normal motorneurons stained deeply; this is a translation to the high amount of

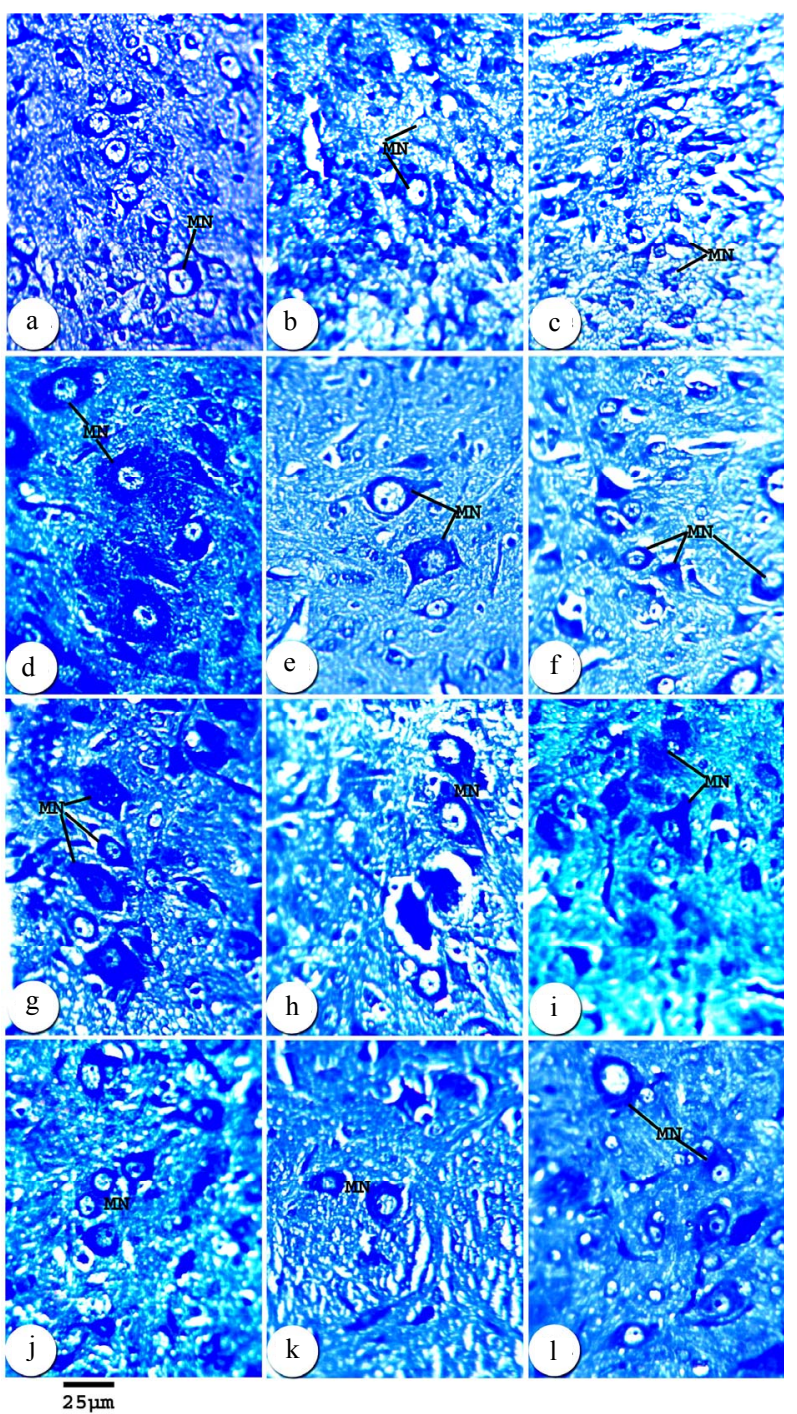

Figure 10. Transverse sections in the brachial spinal region showing the distribution of Nissl granules in motorneurons (MN). (a) Normal group at D7; (b) Group B at D7; (c) Group C at D7; (d) Normal group at D14; (e) Group B at D14, (f) Group $\mathrm{C}$ at D14; (g) Normal group at D21; (h) Group B at D21; (i) NisslGroup C at D21; (j) Normal group at D28; (k) Group B at D28 and (1) Group C at D28 (Toludine-blue stain).
Nissl granules in normal group (Figures 10(d), (g) and (j)). In Groups B and C, motorneurons stained moderately (Figures 10(e), (f), (h), (i), (k) and (l)).

\subsubsection{Feulgen Stain}

The DNA content of brachial motorneuron was demonstrated using Feulgen stain. It was noticed that colour intensity in the normal cells increased with age progress (Figures 11(a), (d), (g) and (j)). The nuclei of Groups B and $\mathrm{C}$ showed a markedly decrease in the colour intensity specific for DNA and central chromatolysis (Figures 11(b), (c), (e), (f), (h), (i), (k) and (l)). Group B showed some improvements at the late ages.

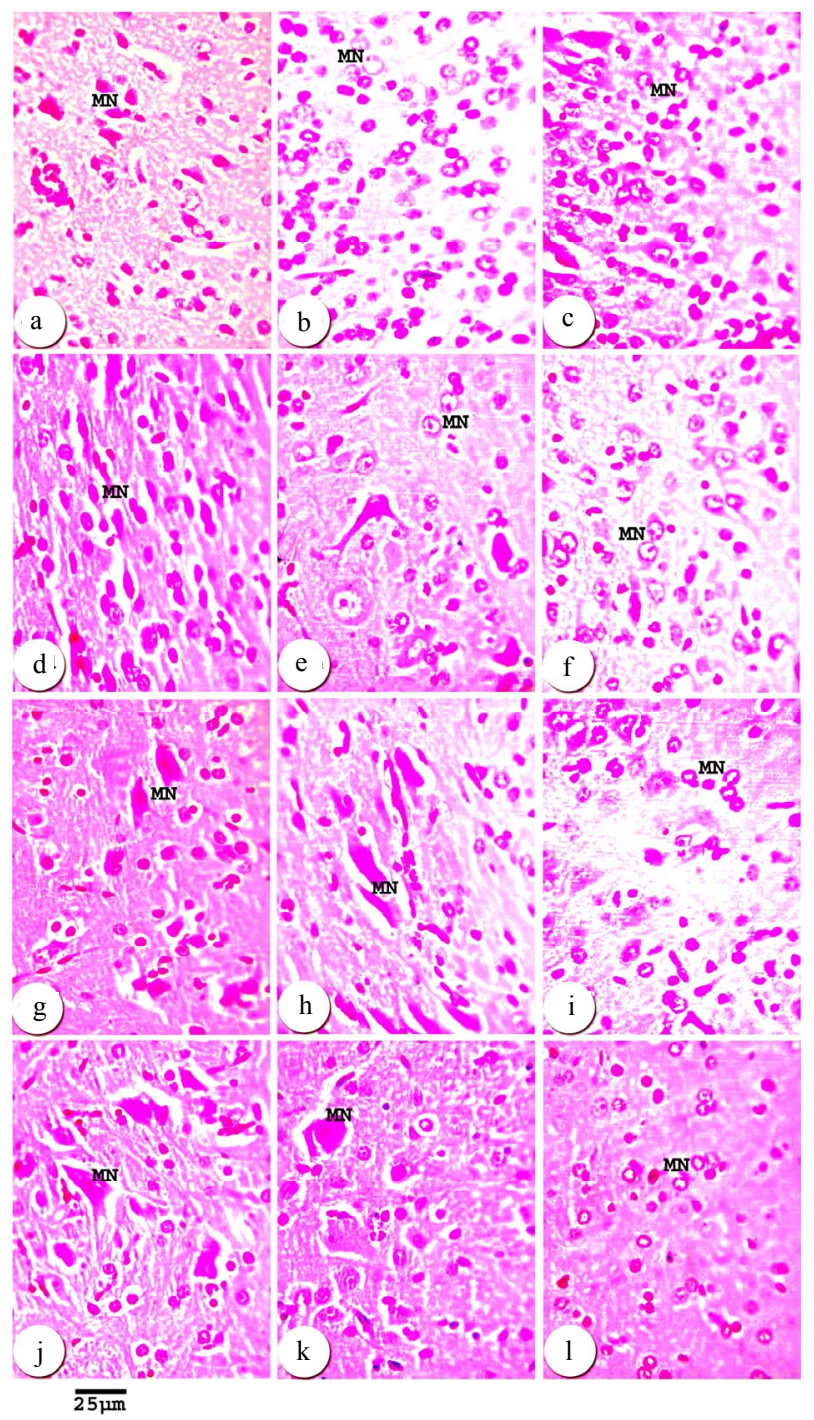

Figure 11. Transverse sections in the brachial spinal region showing DNA in the nuclei of motorneurons (MN). (a) Normal group at D7; (b) Group B at D7; (c) Group C at D7; (d) Normal group at D14; (e) Group B at D14; (f) Group C at D14; (g) Normal group at D21; (h) Group B at D21; (i) Group C at D21; (j) Normal group at D28; (k) Group B at D28 and (1) Group C at D28 (Feulgen staining technique). 


\subsection{The Histopathology and Histochemical Results of Lumber Region of Spinal Cord}

\subsubsection{Haematoxylin-Eosin Stain}

The histological structure of the lumber spinal cord is similar to that of the brachial spinal cord but the difference between both regions depends on the degree of the neuronal development where the lumber motorneurons were relatively smaller than the brachial motorneurons of the same age (Figure 12). At D7, in Groups B and C, most lumber motorneurons appeared small and less differentiated compared to normal ones. Also, pyknosis and neurocyte chromatolysis were detected (Figures 12(a)-

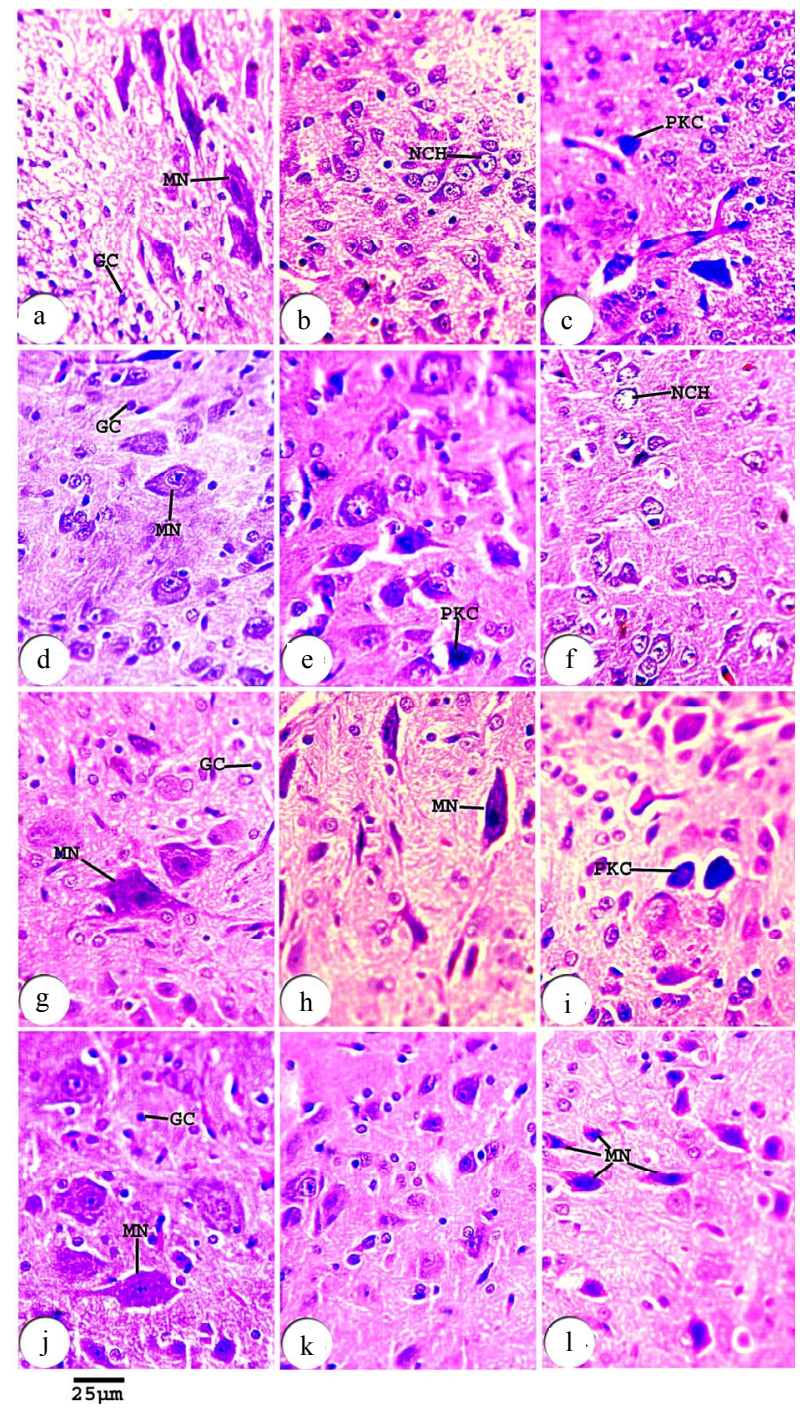

Figure 12. Transverse sections in the lumber spinal region showing the glial cells (GC), motorneurons (MN), neurocyte chromatolysis $(\mathrm{NCH})$ and pyknosis $(\mathrm{PKC})$. (a) Normal group at D7; (b) Group B at D7; (c) Group C at D7; (d) Normal group at D14; (e) Group B at D14; (f) Group C at D14; (g) Normal group at D21; (h) Group B at D21; (i) Group C at D21; (j) Normal group at D28; (k) Group B at D28 and (1) Group C at D28 (H. \& E.). (c)). Between D14 and D28, the normal motorneurons increased in size while pyknotic cells and neurocyte chromatolysis were still represented in treated groups (Figures 12(e), (f), (i) and (l)). Group B showed some improvement at D21 andD28 as in case of brachial region (Figures 12(h) and (k)).

\subsubsection{Toludine-Blue Stain}

Between D7 and D28, the normal lumber motorneurons had a relatively high intensity of Nissl granules as in the brachial motorneurons (Figures 13(a), (d), (g) and (j)). At D7 and D14, motorneurons of Groups B and C were paled stained while at D21 and D28, they were stained moderately (Figures 13(b), (c), (e), (f), (h), (i), (k) and (l)).

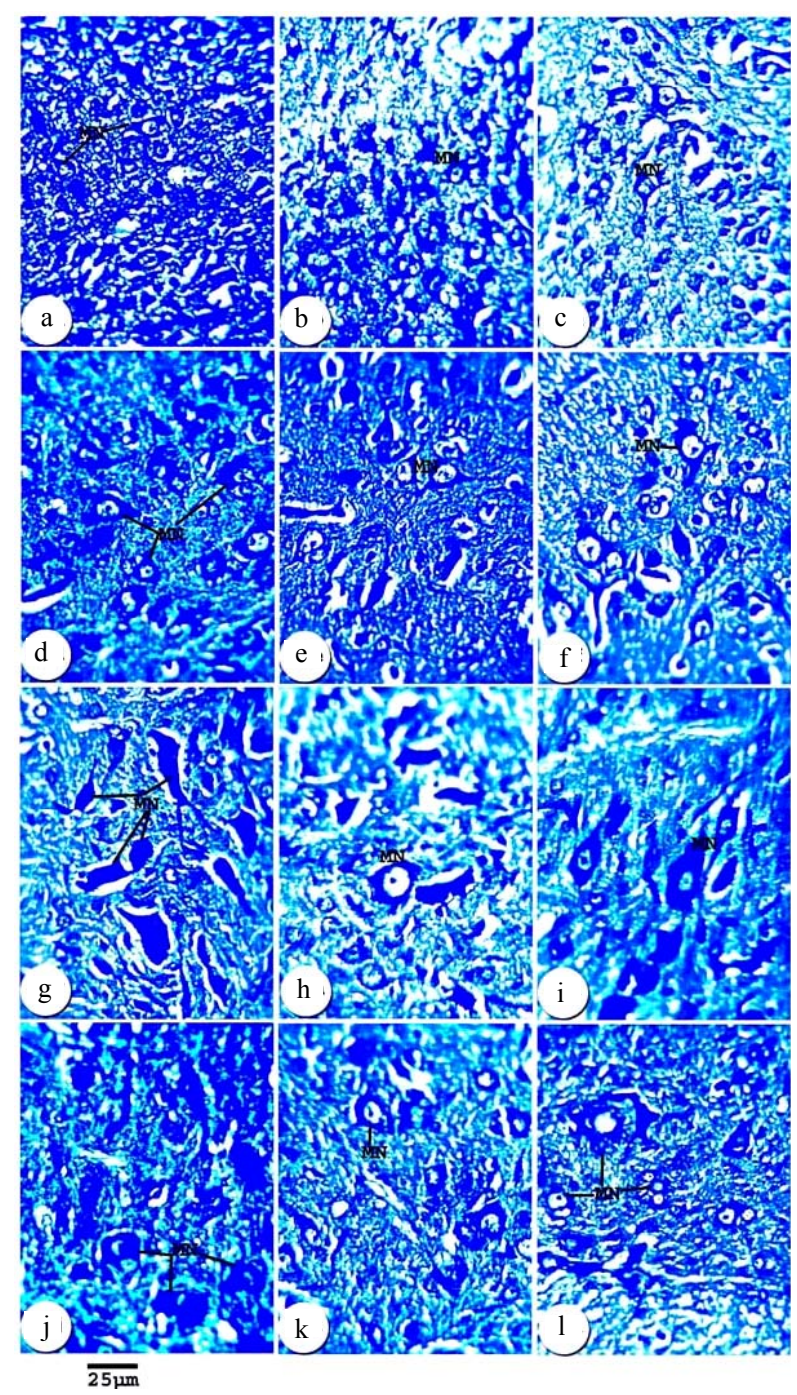

Figure 13. Transverse sections in the lumber spinal region showing the distribution of Nissl granules in motorneurons (MN). (a) Normal group at D7; (b) Group B at D7; (c) Group C at D7; (d) Normal group at D14; (e) Group B at D14; (f) Group C at D14; (g) Normal group at D21; (h) Group B at D21; (i) Group C at D21; (j) Normal group at D28; (k) Group B at D28 and (l) Group C at D28 (Toludine-blue stain). 


\subsubsection{Feulgen Stain}

Feulgen stain showed that the DNA content of normal lumber motorneurons nuclei similar to the case of normal brachial motorneurons but the nuclei of lumber motorneurons were small in size (Figures 14(a), (d), (g) and (j)). In treated groups, it was observed that the colour intensity was markedly decreased as in the brachial region but the DNA-containing particles stainability was less than of brachial ones, especially in Group C (Figures 14(b), (c), (e), (f), (h), (i), (k) and (l)).

\section{DISCUSSION}

\subsection{Sensorimotor Reflexes}

The experimental groups were differed in the develop-

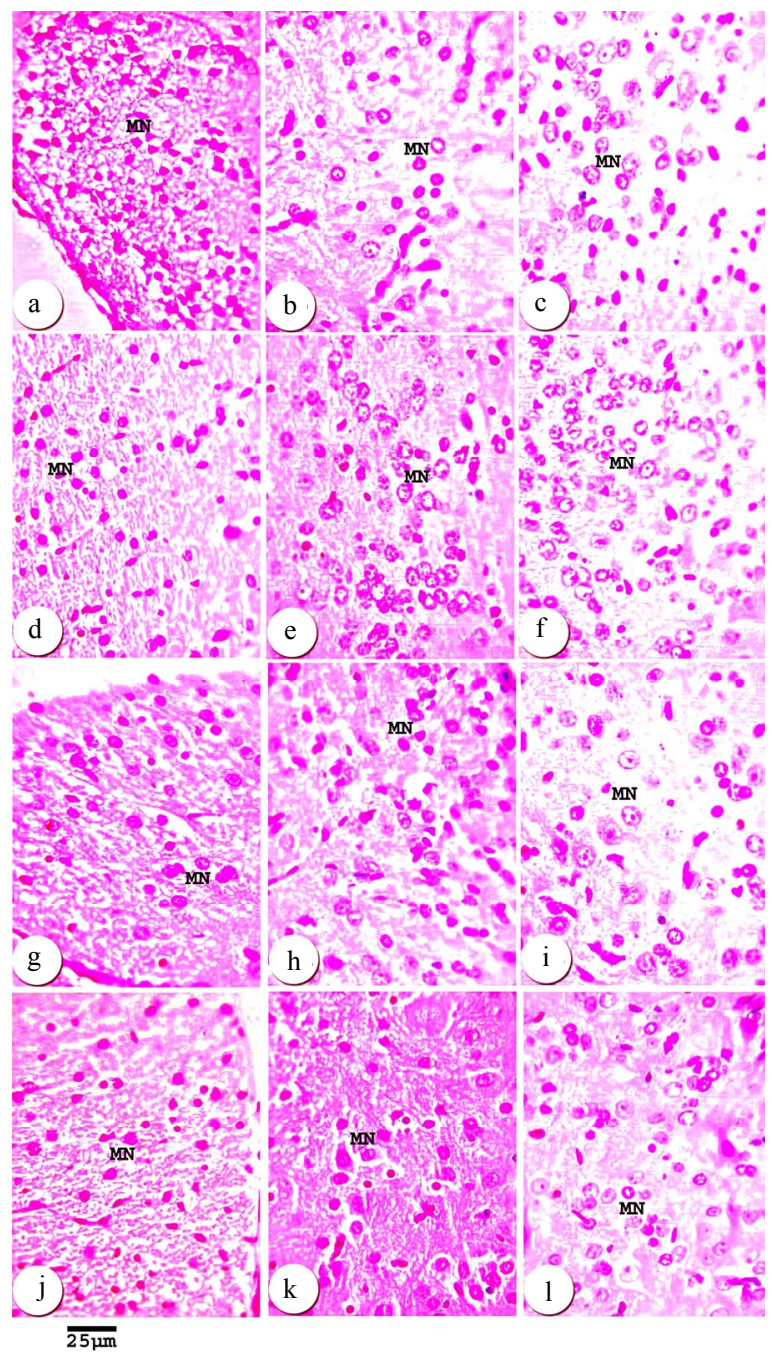

Figure 14. Transverse sections in the lumber spinal region showing DNA in the nuclei of motorneurons (MN). (a) Normal group at D7; (b) Group B at D7; (c) Group C at D7; (d) Normal group at D14; (e) Group B at D14; (f) Group C at D14; (g) Normal group at D21; (h) Group B at D21; (i) Group C at D21; (j) Normal group at D28; (k) Group B at D28 and (1) Group C at D28 (Feulgen staining technique). mental degree of sensorimotor reflexes at the same age. Each reflex reflects the state of a certain part in the CNS and the rate of its development $[10,21,26,31,36]$. In the present treated newborns, the retardation in the development of sensorimotor reflexes is a feature of acrylamide neurotoxicity as mentioned by [25]. Acrylamide exposure leads to prenatal and postnatal malnutrition through its effects on mothers' behaviors [21,37]. [24] recorded that malnutrition causes retardation in the development of sensorimotor reflexes. The present study showed that acrylamide induces neuropathy and neuronal loss which leads to behavioral abnormalities as mentioned by [9,38,39]. [31] recorded that sensorimotor reflexes expression are mediated by the CNS neurons. Therefore, the losing of these neurons will affect the expression of these reflexes. $[40,41]$ reported that the behavioral abnormalities in the developing newborns depend on CNS regions where neuronal loss occurs. Also, acrymide impair synapse function and neuronal connection [28]. It was obvious from the present results that acrylamide causes weakness, ataxia and malformation in the activity of newborns of the treated groups; similar observations were recorded by $[27,42]$.

Rooting reflex had not been elicited in normal and treated groups of rat newborns but the reason was not clear. This result also recorded by [43]. Rooting is innate in mammals [44] because the young find warmth and a nipple. As this need decreases, the reflex also decrease as reported by [23]. The disappearance of rooting in rat pups may be due to inhibition of the subcortical regions responsible for the reflex by the cerebral cortex $[44,45]$. The present study revealed that the lamination of the cerebral cortex is not detectable in rat at D7 and D14 of experimental time.

It is of interest that FL Grasp is a spinal reflex [21,31]. It appeared at $\mathrm{D} 4$ by $31.67 \%$ of the trails in the normal newborns because the neurons-mediated reflexes (motorneurons) differentiated at this age as seen in the present histological sections. The reflex expression takes place after the differentiation of its mediating neurons $[10,18,36]$. In gerbil, this reflex appears after the second postnatal day [31]. In the present normal newborns, the reflex expression was regularly increased to reach $100 \%$ at D10, which indicated the regular rate of cells differentiations. The histological sections showed the well form of the brachial motorneurons. In Group B, this reflex detected at $\mathrm{D} 4$ by $8.33 \%$ due to the weakness of the newborns which resulted from malnutrition regarded to the bad behavior of the mothers as described by [37]. Its development was slow to reach $100 \%$ at D15. In Group C, FL grasp was detected at D5 by $15 \%$ that resulted from retardation in development and differentiation of reflex mediated neurons due to acrylamide exposure. The development of FL grasp reflex in Group C was slow 
and irregular due to less activation of newborns as seen by $[25,46]$. With age progress, this reflex attained $100 \%$ at D17 by the mediating of persisting small neurons. The late appearance and maturation of FL grasp in the newborns of treated groups resulted from malformations in the FL and spinal cord. Also, concerned to acrylamide which leads to malnutrition that delays the appearance and maturation of sensorimotor reflexes [10]. In addition, it was found that acrylamide delays reflex and motor development as reported by $[45,47]$.

HL grasp was observed in normal newborns at D10 by $18.33 \%$ of the trials, which appeared later than the FL grasp. HL grasp was absent in gerbil at all [31], while it present in Brazilian opossum [23]. In normal group, HL grasp developed regularly due to early differentiation of the present spinal cord neurons which mediate this spinal reflex to reach $100 \%$ at D16. The present study showed the high activations of the newborns reflected by the intensity of Nissl granules in cells mediated this reflex as seen by the present histological sections. In Groups B and $\mathrm{C}$, this reflex was detected at D13 by $11.67 \%$ and $6.67 \%$, respectively. After that, the reflex expression increased with the age mediated by the persisting neurons to reach $100 \%$ at D19 in Group B and at D21 in Group C. The present study shows a good relation between intensity of Nissl granules and newborns activation where granules intensity in the neurons refers to the high metabolic activity of these neurons [48]. Therefore, the low activetion of the newborns of the treated groups was reflected by the low intensity of Nissl granules. So acrylamide affects on motor development through its effect on protein synthesis as mentioned by [49]. The late appearance and maturation of the hind-limb grasp reflex resulted from dysfunctions of spinal cord neurons in the treated newborns also this is in agreement with [9]. This results in accordance to acrylamide that delays the motor development $[25,45]$.

Surface body righting reflex is a spinal reflex so it appeared early at D2 in the present normal newborns by $50 \%$ of the trials. This reflex appeared before FL grasp in most mammals except in the Brazilian Opossum [31]. This normal reflex developed rapidly to reach $100 \%$ at D6 that resulted from coordination between FL and HL as well as early maturation of the motorneurons; this is in accordance to $[23,43]$. In Groups B and C, this reflex detected at D2 by $10 \%$ due to acrylamide exposure and developed slowly to reach $100 \%$ at D12 and D13, respectively. The late appearance and maturation were due to weakness of the newborns and malformation in spinal cord. The neuronal loss in spinal cord affects on the spinal reflexes $[10,43,45]$. This retardation in the reflex development in the present treated groups resulted from maternal acrylamide exposure, which not only leads to malnutrition and protein deficiency as reported by [50] but also leads to CNS neuronal loss [10,37], CNS neuropathy [51,52], weakness and ataxia [53] all the above mentions cause retardation in reflexes development.

Air body righting is a cerebellar reflex, it appeared late in normal rat newborns because cerebellum maturation occurs late $[10,31,45]$. This normal reflex detected at D11 by $30 \%$ of the trials and regularly increased to reach $100 \%$ at D17. This pattern of reflex development is due to continuous differentiation and maturation of the present cerebellar neurons. These results go parallel with [54]. It reaches $100 \%$ at D22 in Mongolian gerbil [31] and at D21 in both rabbit and Guinea pig [18]. In Group $\mathrm{B}$, this reflex detected at D15 by $16.67 \%$ and developed to reach $100 \%$ at D20. In Group C, it detected late at D17 by $18.33 \%$ and developed to reach $100 \%$ at D22. This reflex developed earlier in Group B than in Group C because the newborns of Group $\mathrm{C}$ exposed chronically to acrylamide during lactation period. In both treated groups, the retardation in reflex development resulted from the late maturation and differentiation of the cerebellar neurons and from the high number neuronal loss mediated by acrylamide. Similar observations were recorded by several authors [27,25].

FL hopping is a spinal reflex and tested in the forward direction so it was detected early in Mongolian gerbil [31] and in Brazilian opossum [23] due to early maturation of motorneurons in spinal cord $[18,10]$. This reflex detected at D3 in normal rat newborns by $48.33 \%$ and increased rapidly to reach $100 \%$ at D8. This activation in reflex expression is mediated by well-differentiated neurons. This result goes parallel with $[43,55]$. In Groups B and C, it was observed by $13.33 \%$ and $43.33 \%$ at D4 and D5, respectively. The reflex developed slowly and irregularly in both groups to reach $100 \%$ at D14 in Group B and at D15 in Group C. This retardation in reflex development due to maternal acrylamide consumption leads to neuropathy in the CNS of their newborns as reported by [10, $25]$. The late appearance and maturation of this reflex resulted from the malformation in spinal cord and FL functions in the present treated newborns.

HL hopping reflex tested in the forward direction as in the case of FL hopping reflex. It appeared in Brazilian opossum [23] and Mongolian gerbil [36]. In addition, it is a spinal reflex which mediated by motorneurons [31]. So it appeared early in normal group at D3 by $38.33 \%$ and developed rapidly to reach $100 \%$ at D10. The present study showed that HL hopping maturation took place after differentiation of motorneurons, also [36] and [10] reported similar results. This reflex developed by similar manner as in FL hopping in Groups B and C but its maturation was later than in FL hopping in the normal and treated groups. Its maturation detected at D15 in Group B and at D18 in Group C. The late appearance and maturation of this reflex regarded to malformations 
in spinal cord and HL under the effect of acrylamide.

Chin tactile placing reflex is a cerebral reflex [31]. So, it appeared early at $\mathrm{D} 4$ by $8.33 \%$ after spinal reflexes and before cerebellar reflexes, but it takes long time to reach $100 \%$ at D11 in normal rat newborns because the present study showed that cerebral cortex takes long time to be matured after birth in rat newborns. This result is in agreement with $[18,43]$. The development of this reflex is similar in Groups B and C. It appeared at D8 by $21.67 \%$ in Group B and by $8.33 \%$ in Group C. It increased to attain $100 \%$ at D16 in Group B and at D17 in Group C. The late appearance of this reflex is due to the abnormalities in the cerebral cortex of the newborns of these groups [31]. The abnormalities in reflex expression resulted from the cerebral neuropathy caused by acrylamide as reported by [37]. The performance of this reflex requires the involvement of numerous central and peripheral nervous system components. Thus, acrylamide effects on these two tasks may impact a variety of systems and/or regions, such as muscle strength and response to fatigue [25,27].

The visual placing reflex is a cerebral reflex but its expression related to eye opening [31,43]. In normal rat newborns, this reflex expressed at D15 by $46 \%$ and expressed by $100 \%$ at D17. The eye opening completed at D15 so the animal takes a time to do the reflex after eye opening. This result is in agreement with [24]. In the other treated groups this reflex expressed at D16 by $28.33 \%$ and $27 \%$ in Groups B and C, respectively. The previous ratios depend on the number of newborns, which had opened eyes, and able to expressed this reflex physically. It developed to reach $100 \%$ at D19 in Group B and at D21 in Group C. This retardation produced by acrylamide which leads to skeletal muscles weakness and developmental alterations of the offspring $[25,42]$. Also, acrylamide impair synapse function and neuronal connection [28] so it affects on the expression of this reflex.

\subsection{Comparative Histopathological Studies}

The detected spinal cord in normal and treated rat newborns is surrounded with meninges and consists of centrally located gray matter where neurons and glial cells are distributed and peripheral white matter. [36] mentioned that in the Mongolian gerbil at different ages, the motorneurons of the ventral horn of spinal cord are already well differentiated in the day of birth, but the neurons of the dorsal horn are smaller, less differentiated and more densely packed, these findings agree with those of $[18,56]$ on rat newborns.

In the present normal rat newborns, the brachial and lumber motorneurons were well differentiated at D7. Their number and size increased with age progress, so the spinal reflexes appeared and mature early after neurons maturation. The brachial motor neurons appeared more differentiated than the lumber neurons. Also, [18] reported that the brachial motorneurons are larger in size and number than in the lumber motorneurons. The neurotransmitters, like serotonin and noradrenaline, are supposed to play a crucial role in the differentiation and proliferation of the spinal cells $[10,57]$.

In the present treated groups, the motorneurons were small less differentiated compared to normal ones. Between D7 and D28, the normal motorneurons increased in size, while pyknosis and neurocyte chromatolysis were observed in the treated groups due to chronic acrylamide exposure. Group B showed some improvement at D21 and D28 because of absence of acrylamide during lactation. The present results improved that acrylamide ingestion by pregnant dams will cause cells loss during the development as reported by $[37,53]$. Acrylamide exposure reduced motorneurons functions $[53,45]$. Acrylamide consumption impairs motor coordination, motor control and behavioral activities that are regulated by functional integration of neuronal activity in various regions of the brain and the spinal cord. It reduced motorneurons ability to generate action potentials $[45,47,58]$. Also, it effects on the pattern of neuronal axons and fibers as well as synapse [9]. Therefore, acrylamide induced dysfunction of neuronal electrical activity in the mammalian spinal cord.

\subsection{Histochemical Studies}

The intensity of Nissl granules in the neurons refers to the high metabolic activity of these neurons [48]. In Group A of the present study, the intensity of Nissl granules in spinal cord neurons was high at all investigation stages, which indicate the high rate of metabolism in newborns neurons of this group. The present study showed that the intensity of Nissl granules in the neurons were more compact and have thickened cytoplasm coat. These evidences are in agreement with [48] who found the necessary protein so-called Nissl granules are present in the cell body and dendrites.

In Group C, the investigated neurons appeared faint in all stages due to the low amount of intracellular Nissl granules and proteins. This result indicates the low rate of metabolism in the neurons of this group, which may affect on cells functions and in turn affect on the activation of the newborns. In Group B, the cells appeared paled at D7 and D14 due to the low amount of Nissl granules and proteins but stained intermediated at D21 and D28, which reflected the slow improvement due to acrylamide withdrawals. The Nissl granules intensity in treated groups was low due to the inability of the fetus to synthesize required proteins where Nissl granules in neurons are essential protein $[45,48]$. Acrylamide directly impairs protein synthesis in fetal tissues through its effect on RNA and DNA as well as total and subcel- 
lular protein contents [59]. There are other metabolic effects of acrylamide on the cell function include formation of free radical, alteration of cytoskeleton elements, cells membrane necrosis and mitochondrial destruction $[49,60]$. Also, acrylamide indirectly affects on the protein synthesis because it resulted in nutrients deficiency which are essential in protein synthesis due to pre- and post-natal acrylamide exposure impair the functions of placenta and mammary glands [10,37,61]. The above factors collectively reduce the metabolic activities of cell and protein synthesis due to inadequate nutrients so the intensity of Nissl granules was low in the treated group. This explain the reduction in the activity of the treated newborns which supported by the results of sensorimotor reflexes.

DNA in the investigated neurons was stained red to pink using Feulgen stain. The present histochemical result revealed that colour intensity in the normal cells increased with age progress reflected the increasing in DNA content of the cells with age development till D28 due to the change in cells size. The nuclei of Groups B and $\mathrm{C}$ showed a markedly decrease in the intensity of the colour specific for DNA demonstration, indicating a marked loss of DNA. This colour reduction decreased with age development in Group B but increased in Group C. Moreover, central chromatolysis that observed in most ages of the present treated groups due to DNA damage induced by acrylamide $[45,62,63]$. The present study showed chromatolysis in the most neurons of the developmental rat newborns due to acrylamide and its metabolic glycidamide derivative, which have a high potential to form DNA adducts through their reaction with DNA $[10,59,64]$. In Chinese hamster lung cell lines, acrylamide induced alteration in cell division and chromosomal aberrations [65].

In conclusion, acrylamide affects on CNS regions of the developed rat newborns if their mothers exposed to acrylamide during gestation and lactation periods. These effects appeared as histopathological changes within the newborns tissues. These disturbances in the newborns lead to behavioral and healthy abnormalities. So, acrylamide has a teratogenic effect on the rat newborns.

\section{REFERENCES}

[1] Friedman, M. (2003) Chemistry, biochemistry, and safety of acrylamide. A review. Journal of Agricultural and Food Chemistry, 51, 4504-4526. doi:10.1021/jf030204+

[2] Dixit, R., Seth, P.K. and Mukjtar, H. (1982) Metabolism of acrylamide into urinary mercapturic acid and cysteine conjugates in rats. Drug Metabolism and Disposition, 10, 196-197.

[3] Miller, M.J., Carter, D.E. and Sipes, I.G. (1982) Pharmacokinetics of acrylamide in Fisher-334 rats. Toxicology and Applied Pharmacology, 63, 36-44.
doi:10.1016/0041-008X(82)90024-2

[4] Calleman, C.J., Bergmark, E. and Costa, L. (1990) Acrylamide is metabolized to glycidamide in the rat, evidence from hemoglobin adducts formation. Chemical Research in Toxicology, 3, 406-412. doi:10.1021/tx00017a004

[5] Sumner, S.C.J., MacNeela, J.P. and Fennell, T.R. (1992) Characterization and quantitation of urinary metabolites of [1,2,3-13C]acrylamide in rats and mice using carbon13 nuclear magnetic resonance spectroscopy. Chemical Research in Toxicology, 5, 81-89. doi: $10.1021 / \mathrm{tx} 00025 \mathrm{a} 014$

[6] Das, M., Mukhtar, H. and Seth, P. (1982) Effect of acrylamide on brain and hepatic mixed function oxidases. Toxicology and Applied Pharmacology, 66, 420-426. doi:10.1016/0041-008X(82)90308-8

[7] Sumner, S.C.J., Selvaraj, L. and Nauhaus, S.K. (1997) Urinary metabolites from $\mathrm{F} 344$ rats and $\mathrm{B} 6 \mathrm{C} 3 \mathrm{~F} 1$ mice coadministered acrylamide and acrylonitrile for 1 or 5 days. Chemical Research in Toxicology, 10, 1152-1160. doi:10.1021/tx9602123

[8] Spencer, P.S. and Schaumburg, H.H. (1979) Clinical and experimental studies of distal axonopathy, a frequent form of brain and nerve damage produced by environmental chemical hazards. Annals of the New York Academy of Sciences, 329, 14-29. doi:10.1111/j.1749-6632.1979.tb15331.x

[9] LoPachin, R.M. (2004) The changing view of acrylamide neurotoxicity. Neurotoxicology, 25, 617-630. doi:10.1016/j.neuro.2004.01.004

[10] Allam, A., El-Ghareeb, A., Abdul-Hamid, M., Baikry, A. and Sabri, M. (2011) Prenatal and perinatal acrylamide disrupts the development of cerebellum in rat, biochemical and morphological studies. Toxicology and Industrial Health, 27, 291-306. doi:10.1177/0748233710386412

[11] Takahashi, S., Tanaka, H. and Oki, J. (1999) Development of spinal motorneurons in rats after aneonatal hypoxic insult. Pediatric Neurology, 21, 715-720. doi:10.1016/S0887-8994(99)00080-6

[12] Delcomyn, F. (1980) Neural basis of rhythmic behavior in animals. Science, 210, 492-498. doi:10.1126/science.7423199

[13] Grillner, S. (1985) Neurobiological bases of rhythmic motor acts in vertebrates. Science, 228, 143-149. doi:10.1126/science.3975635

[14] Sakurai, M., Tayashi, H., Abe, K., Itoyama, Y. and Tabayashi, K. (2000) Cyclin D1 and Cdk4 protein induction in motor neurons after transient spinal cord ischemia in rabbits. Stroke, 31, 200-207. doi:10.1161/01.STR.31.1.200

[15] Sinkjaer, T., Toft, E. and Hansen, H.J. (1995) H-reflex modulation during gait in multiple sclerosis patients with spasticity. Acta Neurologica Scandinavica, 91, 239-264. doi:10.1111/j.1600-0404.1995.tb06997.x

[16] Dubowitz, V. (1995) Musle disorders of childhood. W.B. Saunders, Co. Ltd., London.

[17] Williams, B.Y., Vinnakota, S., Sawyer, C.A., Woldrep, J.C., Hamilton, S.L. and Sarkar, H.K. (1999) Differential subcellular localization of the survival motor neuron pro- 
tein in spinal cord and skeletal muscle. Biochemical and Biophysical Research Communications, 254, 10-14.

[18] Dalia, M.S. (2002) Comparative studies on the ontogeny of sensorimotor reflexes and locomotive activity in small mammals and their applications on infants. Ph.D. Thesis, Mansour University, Mansour.

[19] Zehr, E.P. and Stein, R.B. (1999) What functions do reflexes serve during human locomotion. Progress in $\mathrm{Neu}-$ robiology, 58, 185-205. doi:10.1016/S0301-0082(98)00081-1

[20] Rossignol, S. (1996) Neural control of stereotypic limb movement. In: Rowell, L.B. and Sheperd, J.T., Eds., Integration of Motor Circulatory, Respiretory and Metabolic Control during Exercise, Oxford University Press, New York, 173-216.

[21] Seale, S.M., Feng, Q., Agarwal, A.K and El-Alfy, A.T. (2012) Neurobehavioral and transcriptional effects of acrylamide in juvenile rats. Pharmacology Biochemistry and Behavior, 1, 84.

[22] Nicholls, J.G., Martin, A.R. and Wallace, B.G. (1992) From neuron to brain. 3rd Editon, Sinauer Associates Inc., Sunderland.

[23] Cassidy, G., Boudrias, D., Pflieger, J.F. and Cabana, T. (1994) The development of sensorimotor reflexes in the Brazilian opossum (Monodelphis domestica). Brain, Behavior and Evolution, 43, 244-253. doi:10.1159/000113638

[24] Smart, J.L. and Dobbing, J. (1971) Vulnerability of developing brain. II. Effects of early nutritional deprivation on reflex ontogeny and development of behaviour in the rat. Brain Research, 28, 85-95. doi:10.1016/0006-8993(71)90526-9

[25] Garey, J., Sherry, A.F. and Merle, G.P. (2005) Developmental and behavioral effects of acrylamide in Fischer 344 rats. Neurotoxicology and Teratology, 27, 3-563. doi:10.1016/j.ntt.2005.03.007

[26] Zhang, L., Gavin, T., Barber, D.S. and LoPachin, R.M. (2011) Role of the Nrf2-ARE pathway in acrylamide neurotoxicity. Toxicology Letters, 5, 7-11. doi:10.1016/j.toxlet.2011.08.017

[27] Jennekens, F.G., Veldman, H., Schotman, P. and Gispen, W.H. (1979) Sequence of motor nerve terminal involvement in acrylamide neuropathy. Acta Neuropathologica, 46, 57-63. doi:10.1007/BF00684805

[28] Agrawal, A.K. and Squibb, R.E. (1981) Effects of acrylamide given during gestation on dopamine receptor binding in rat pups. Toxicology Letters, 7, 233-238. doi:10.1016/0378-4274(81)90074-6cvc

[29] Kinnard, W.J. and Watzman, N. (1966) Techniques used in the evaluation of psychotropic drugs on animal activity. Journal of Pharmaceutical Sciences, 55, 995-1012. doi:10.1002/jps.2600551002

[30] Bouet, V., Wubbels, R.J., De-Jong, H.A.A. and Gramsbergen, A. (2004) Behavioral consequences of hypergravity in developing rats. Developmental Brain Research, 153, 69-78. doi:10.1016/j.devbrainres.2004.03.022

[31] Cassidy, G., Pflieger, J.F. and Cabana, T., (1992) The on- togenesis of sensorimotor reflexes in the Mongolian gerbil (Meriones unguicatas). Behavioural Brain Research, 52, 143-151. doi:10.1016/S0166-4328(05)80224-4

[32] Mallory, F.B. (1988) Pathological Techénique. W.B. Saunders, Philadelphia.

[33] Feulgen, R. and Rossenbeck, H. (1924) Mikroskopischchemischer Nachweis einer Nucleinsäure vom Typus der Thymonucleinsäure und die-darauf beruhende elektive Färbung von Zellkernen in mikroskopischen Präparat. Zeitschrift für Physikalische Chemie, 135, 203-248. doi:10.1515/bchm2.1924.135.5-6.203

[34] Carleton, H., Drury, R., Willington, E. and Conergon, H. (1967) Cited from Carleton. Histological techniques. Oxford University Press, Toronto.

[35] Rao, M. and Blane, K. (1995) PC-STAT. One way analysis of variance procedure. University of Georgia, Atlanta.

[36] Cabana, T., Cassidy, G., Pflieger, J.F. and Baron, G. (1993) The ontogenic development of sensorimotor reflexes and spontaneous locomotion in the Mongolian gerbil (Meriones unguicutas). Brain Research Bulletin, 30, 291-301. doi:10.1016/0361-9230(93)90257-C

[37] Shaheed, I.B., Kawkab, A.A. and Makhlouf, M.M. (2006) Toxicicological and pathological studies on acrylamide neurotoxicity in albino rats. Egyptian Veterinary Medical Society for Pathology and Clinical Pathology, 19, 63-82.

[38] Crofton, K.M., Padilla, S., Tilson, H.A., Anthony, D.C., Raymer, J.H. and MacPhail, R.C. (1996) The impact of dose rate on the neurotoxicity of acrylamide, the interacttion of administered dose, target tissue concentrations, tissue damage, and functional effects. Toxicology and Applied Pharmacology, 139, 163-176. doi:10.1006/taap.1996.0155

[39] Lehning, E.J., Balaban, C.D., Ross, J.F., Reid, M.A. and LoPachin, R.M. (2002) Acrylamide neuropathy. I. Spatiotemporal characteristics of nerve cell damage in rat cerebellum. Neurotoxicology, 23, 397-416. doi:10.1016/S0161-813X(02)00083-9

[40] Goodlett, C.R., Marcussen, B.L. and West, J.R. (1992) A single day of alcohol exposure during the brain growth spurt induce brain weight restriction and cerebellar Purkinje cell loss. Alcohol, 7, 107-114. doi:10.1016/0741-8329(90)90070-S

[41] Daniel, J.B., Nancy, E.B., Ruth, M.A.N., Susan, J.A. and West, J.R. (1996) Purkinje cell deficits in nonhuman primates following weekly exposure to ethanol during gestation. Teratology, 53, 230-236. doi:10.1002/(SICI)1096-9926(199604)53:4<230::AID-T ERA5>3.0.CO;2-6

[42] LoPachin, R.M., Ross, J.F., Reid, M.L., Dasgupta, S., Mansukhani, S. and Lehning, E.J. (2002) Neurological evaluation of toxic axonopathies in rats, acrylamide and 2,5-hexanedione. Neurotoxicology, 23, 95-110. doi:10.1016/S0161-813X(02)00003-7

[43] Abdul-Hamid, M., Allam, A. and Hussein, M.B. (2007) Effect of ethanol administration during gestation on the cerebral cortex and spinal cord of albino rat newborns and on the development of their sensorimotor reflexes. Egyptian Journal of Zoology, 48, 137-162.

[44] Fox, M.W. (1964) A phylogenetic analysis of behavioral 
neuro-ontogeny in precocial and non-precocial mammals. Canadian Journal of Comparative Medicine and Veterinary Science, 28, 197-202.

[45] Guo, C., Li, B. and Xiao, J. (2010) General survey of mechanisms of acrylamide neurotoxicity. Journal of $\mathrm{Hy}$ giene Research, 39, 282-285.

[46] Garey, J. and Paule, M.G. (2010) Effects of chronic oral acrylamide exposure on incremental repeated acquisition (learning) task performance in Fischer 344 rats. Neurotoxicology and Teratology, 32, 20-25. doi:10.1016/j.ntt.2009.10.001

[47] Wise, L.D., Gordon, L.R., Soper, K.A., Duchai, D.M. and Morrissey, R.E. (1995) Developmental neurotoxicity evaluation of acrylamide in Sprague-Dawley rats. Neurotoxicology and Teratology, 17, 189-198. doi:10.1016/0892-0362(94)00071-K

[48] Stevens, A. and Lowe, J. (1997) Human Histology. 2nd Edition, Grafos S.A., Barcelona.

[49] Sridevi, B., Reddy, K.V. and Reddy, S.L.N. (1998) Effect of trivalent and hexavalent chromium on antioxidant enzyme activities and lipid peroxidation in a freshwater field crab, Barytelphusa guerini. Bulletin of Environmental Contamination and Toxicology, 61, 384-390. doi:10.1007/s001289900774

[50] Frieda, S.G. and William, P.R. (1999) Effects of lactational administration of acrylamide on rat dams and offspring. Reproductive Toxicology, 13, 511-520. doi:10.1016/S0890-6238(99)00043-X

[51] LoPachin, R.M., Lehning, E.J., Opanashuk, L.A. and Jortner, B.S. (2000) Rate of neurotoxicant exposure determines morphologic manifestations of distal axonopathy. Toxicology and Applied Pharmacology, 167, 75-86. doi:10.1006/taap.2000.8984

[52] Lehning, E.J., Balaban, C.D., Ross, J.F. and LoPachin, R.M. (2003) Acrylamide neuropathy, III. Spatiotemporal charcteristics of nerve cell damage in forebrain. Neurotoxicology, 24, 125-136. doi:10.1016/S0161-813X(02)00155-9

[53] Ko, M.H., Chen, W.P., Linshiau, S.Y. and Hsieh, S.T. (1999) Age-dependent acrylamide neurotoxicity in mice, morphology, physiology and function. Experimental Neurology, 158, 37-46. doi:10.1006/exnr.1999.7102

[54] Laouris, Y., Kalli-Laouris, J. and Schwartze, P. (1990) The influence of altered head, thorax and pelvis mass on the postnatal development of air-righting reaction in albino rats. Behavioural Brain Research, 38, 185-190. doi:10.1016/0166-4328(90)90016-8

[55] Stelzner, D.J. (1971) The normal postnatal development of synaptic end-feet in the lumbosacral spinal cord and responses in the hind limbs of the albino rat. Experimental Neurology, 31, 337-357. doi:10.1016/0014-4886(71)90237-8

[56] Tanaka, H., Takahashi, S. and Oki, J. (1997) Developmental regulation of spinal motorneurons by monoaminergic nerve fibers. Urnal of the Peripheral Nervous System, 2, 323-332.

[57] Rajaoftra, N., Sandillon, F., Geffard, M. and Privat, A. (1989) Pre- and post-natal ontogeny of serotonergic projections to the rat spinal cord. Journal of Neuronscience Research, 22, 305-321.

[58] Lehning, E.J., Balaban, C.D., Ross, J.F. and LoPachin, R.M. (2003) Acrylamide neuropathy, II. Spatiotemporal charcteristics of nerve cell damage in brainstem and spinal cord. Neurotoxicology, 24, 109-123. doi:10.1016/S0161-813X(02)00192-4

[59] Klaunig, J.E. and Kamendulis, L.M. (2005) Mechanism of acrylamide induced rodent carcinogensis. Advances in Experimental Medicine and Biology, 561, 49-62. doi:10.1007/0-387-24980-X 4

[60] Yousef, M.I. and El-Demerdash, F.M. (2006) Acrylamide-induced oxidative stress and biochemical perturbations in rats. Toxicology, 219, 133-141. doi:10.1016/j.tox.2005.11.008

[61] Sorgel, F., Weissenbacher, R., Kinzig-Schippers, M., Hofmann, A., Illauer, M., Skott, A. and Landersdorfer, C. (2002) Acrylamide, increased concen-trations in homemade food and first evidence of its variable absorption from food, variable metabolism and placental and breast milk transfer in humans. Chemotherapy, 48, 267-274. doi:10.1159/000069715

[62] Tyl, R.W., Marr, M.C., Myers, C.B., Ross, W.P. and Friedman, M.A. (2000) Relationship between acrylamide reproductive and neurotoxicity in male rats. Reproductive Toxicology, 14, 147-57. doi:10.1016/S0890-6238(00)00066-6

[63] Tyl, R.W., Friedman, M.A., Losco, P.E. and Ross, W.P. (2000) Rat two-generation reproduction and dominant lethal study of acrylamide in drinking water. Reproductive Toxicology, 14, 385-401. doi:10.1002/em.2850160302

[64] Sega, G.A., Generoso, E. and Brimer, P. (1990) Acrylamide exposure delayed unscheduled DNA synthesis in germ cells of mal mice that is correlated with the temproal pattern of adduct formation in testis DNA. Environmental and Molecular Mutagenesis, 16, 1161-1165.

[65] Warr, T., Parry, J. and Callander, R. (1990) Methyl vinyl sulphone, a new class of Michael-type genotoxin. Mutation Research, 245, 191-199. doi:10.1016/0165-7992(90)90049-P 